\title{
LOCAL WELL-POSEDNESS OF PRANDTL EQUATIONS FOR COMPRESSIBLE FLOW IN TWO SPACE VARIABLES
}

\author{
Ya-Guang Wang, Feng Xie \\ Department of Mathematics, and LSC-MOE, \\ Shanghai Jiao Tong University, Shanghai 200240, P.R.China \\ Tong Yang \\ Department of Mathematics, City University of Hong Kong, \\ Tat Chee Avenue, Kowloon, Hong Kong
}

\begin{abstract}
In this paper, we consider the local well-posedness of the Prandtl boundary layer equations that describe the behavior of boundary layer in the small viscosity limit of the compressible isentropic Navier-Stokes equations with non-slip boundary condition. Under the strictly monotonic assumption on the tangential velocity in the normal variable, we apply the NashMoser-Hörmander iteration scheme and further develop the energy method introduced in [1] to obtain the well-posedness of the equations locally in time.
\end{abstract}

2000 Mathematical Subject Classification: 35A07, 35G61, 35K65.

Keywords: Compressible Prandtl layer equations, monotonic velocity field, energy method, Nash-Moser-Hörmander iteration, local well-posedness.

\section{Introduction}

In this paper, we study the well-posedness of the compressible Prandtl boundary layer equations that are derived in the small viscosity limit from the compressible isentropic Naiver-Stokes equations with non-slip boundary condition. Note that the Prandtl equations describe the behavior of the characteristic boundary layer in the leading order. Denote by $\mathbb{T} \times \mathbb{R}^{+}=\{(x, \eta) \mid x \in$ $\mathbb{R} / \mathbb{Z}, 0 \leq \eta<+\infty\}$ the periodic spatial domain, and let $u(t, x, \eta)$ and $v(t, x, \eta)$ be the tangential and normal velocity components in the boundary layer. Consider the following compressible Prandtl equations with $(x, \eta) \in \mathbb{T} \times \mathbb{R}^{+}$,

$$
\left\{\begin{array}{l}
u_{t}+u u_{x}+v u_{\eta}-\frac{1}{\bar{\rho}(t, x)} \partial_{\eta}^{2} u+P_{x}=0 \\
\partial_{x}(\bar{\rho} u)+\partial_{\eta}(\bar{\rho} v)=-\bar{\rho}_{t}
\end{array}\right.
$$

\footnotetext{
$2 \quad$ E-mail address: ygwang@sjtu.edu.cn(Y.G. Wang), tzxief@sjtu.edu.cn (F. Xie), matyang@cityu.edu.hk(T. Yang)
} 
with the initial data

$$
\left.u(t, x, \eta)\right|_{t=0}=u_{0}(x, \eta),
$$

and the boundary and the far-field conditions

$$
\left.u(t, x, \eta)\right|_{\eta=0}=0,\left.\quad v(t, x, \eta)\right|_{\eta=0}=0, \quad \lim _{\eta \rightarrow+\infty} u(t, x, \eta)=U(t, x) .
$$

Here, $\bar{\rho}(t, x)$ and $U(t, x)$ are the traces on the boundary $\{y=0\}$ of the density and the tangential velocity of the outer Euler flow that satisfy the Bernoulli's law

$$
U_{t}+U U_{x}+P_{x}=0
$$

with $P(t, x)$ being the trace of the enthalpy of the outer Euler flow.

It is well-known that the leading order characteristic boundary layer for the incompressible Navier-Stokes equations with non-slip boundary condition is described by the classical Prandtl equations that were proposed by Prandtl [17] in 1904. Under the monotone assumption on the tangential velocity in the normal direction, Oleinik firstly obtained the local existence of classical solutions in the two spatial dimension by using the Crocco transformation, cf. [15]. This result together with some other extensions in this direction are presented in Oleinik-Samokhin's classical book [16]. Recently, this well-posedness result was re-proved by using an energy method in the framework of Sobolev spaces in [1] and [1] independently. On the other hand, by imposing an additional favorable condition on the pressure, a global in time weak solution was obtained in 22 .

When the monotonicity condition is violated, seperation of the boundary layer is well expected and observed. For this, E-Engquist constructed a finite time blowup solution to the Prandtl equations in [4]. Recently, when the background shear flow has a non-degenerate critical point, some interesting ill-posedness (or instability) phenomena of solutions to both the linear and nonlinear Prandtl equations around the shear flow are studied, cf. [5, 6, 7, 8, All these results show that the monotone assumption on the tangential velocity is very important for well-posedness except in the framework of analytic functions studied in [2] and some other references with generalization.

This paper aims to obtain the local well-posedness of the problem (1.1)-(1.3) for the compressible Prandtl equations in some weighted Sobolev spaces. To state the main results, we first give the following assumptions on the initial data.

\section{Main assumptions $(\mathbf{H})$ on the initial data:}

(H1) For a fixed integer $k_{0} \geq 9$, the initial data $u_{0}(x, \eta)$ satisfies the compatibility condition of the problem (1.1)-(1.3) up to order $4 k_{0}+2$;

(H2) Monotone condition $\partial_{\eta} u_{0}(x, \eta) \geq \frac{\sigma_{0}}{(1+\eta)^{\gamma+2}}>0$ holds for all $x \in \mathbb{T}$ and $\eta \geq 0$ with some positive constant $\sigma_{0}$ and a positive integer $\gamma \geq 2$;

(H3) $\left\|(1+\eta)^{\gamma+\alpha_{2}} D^{\alpha}\left(u_{0}(x, \eta)-U(0, x)\right)\right\|_{L^{2}\left(\mathbb{T} \times \mathbb{R}^{+}\right)} \leq C_{0}$, where $D^{\alpha}=\partial_{x}^{\alpha_{1}} \partial_{\eta}^{\alpha_{2}}$ with $\alpha=\left(\alpha_{1}, \alpha_{2}\right)$ and $|\alpha|=\alpha_{1}+\alpha_{2} \leq 4 k_{0}+2$ 
$(\mathrm{H} 4)$

$\left\|(1+\eta)^{\gamma+2+\alpha_{2}} D^{\alpha} \partial_{\eta} u_{0}\right\|_{L^{\infty}\left(\mathbb{T} \times \mathbb{R}^{+}\right)} \leq \frac{1}{\sigma_{0}}$, for $|\alpha| \leq 3 k_{0}$.

Denote by $V(t, x)$ the trace of $\partial_{y} u_{2}^{E}$ on $\{y=0\}$ for the normal velocity $u_{2}^{E}$ of Euler outer flow. From the conservation of mass in the Euler equations, we have

$$
\partial_{t} \bar{\rho}(t, x)+\partial_{x}(\bar{\rho}(t, x) U(t, x))+\bar{\rho}(t, x) V(t, x)=0 .
$$

Here, we have used the fact that $\left.u_{2}^{E}(t, x, y)\right|_{y=0}=0$. Thus, from the problem (1.1)-(1.3), we know that the normal velocity $v(t, x, \eta)$ can be represented by

$$
v(t, x, \eta)=V(t, x) \eta+\frac{1}{\bar{\rho}(t, x)} \int_{0}^{\eta} \partial_{x}(\bar{\rho}(t, x)(U(t, x)-u(t, x, \tilde{\eta}))) d \tilde{\eta} .
$$

The main result of this paper can be stated as follows.

Theorem 1.1 Suppose that the outer Euler flow is smooth for $0 \leq t \leq T_{0}$, the density $\bar{\rho}(t, x)$ has both positive lower and upper bounds, and the Sobolev norm $H^{s}\left(\left[0, T_{0}\right] \times \mathbb{R}\right)$ of $(\bar{\rho}, U, V)$ is bounded for a suitably large integer s, moreover, the Main Assumption (H) on the initial data $u_{0}(x, \eta)$ is satisfied. Then there exists $0<T \leq T_{0}$, such that the initial boundary value problem (1.1)-(1.3) has a unique classical solutions $(u, v)$ satisfying

$$
\sum_{\left|m_{1}\right|+\left[\left(m_{2}+1\right) / 2\right] \leq k_{0}}\left\|\langle\eta\rangle^{l} \partial_{(t, x)}^{m_{1}} \partial_{\eta}^{m_{2}}(u-U)\right\|_{L^{2}\left([0, T] \times \mathbb{T} \times \mathbb{R}^{+}\right)}<+\infty
$$

for a fixed $l>\frac{1}{2}$ depending only on $\gamma$ given in $(H)$ with $\langle\eta\rangle=(1+\eta)$, and

$$
\sum_{\left|m_{1}\right|+\left[\left(m_{2}+1\right) / 2\right] \leq k_{0}-1} \sup _{\eta \in \mathbb{R}^{+}}\left\|\partial_{(t, x)}^{m_{1}} \partial_{\eta}^{m_{2}}(v-V \eta)(\cdot, \eta)\right\|_{L^{2}([0, T] \times \mathbb{T})}<+\infty .
$$

Remark 1.1 (1) When the outer Euler flow density $\bar{\rho}(t, x)$ is a positive constant, the system (1.1) is reduced to the classical incompressible Prandtl equations. Thus the analysis in this paper works also for the classical incompressible Prandtl equations with general far-field condition and initial data satisfying the Main Assumption (H). Note that the case with a uniform outer flow with slightly different assumption on the initial data was studied in [1].

(2) It is straightforward to verify that the set of the initial data satisfying the Main Assumption (H) is not empty because it contains the functions with polynomial decay in $\eta$.

Now, let us give some comments on the analysis in this paper. In principle, we will apply the approach of 1 . to study the problem (1.1)-(1.3). There are several crucial differences between the system (1.1) and classical incompressible Prandtl equations. Firstly, the normal velocity $v$ contains the linearly increasing part $V(t, x) \eta$ in $\eta$, consequently, in estimating the solution to the linearized problem, we need to study the conormal estimates. Secondly, the divergence free condition in the classical Prandtl system is now replaced by an inhomogeneous equation in (1.1). Moreover, the far-field state is not uniform so that the shear flow is no longer an exact solution to the compressible Prandtl equations (1.1). Therefore, to apply the Nash-MoserHömander iteration scheme used in [1] for the nonlinear problem (1.1)-(1.3), we need to construct 
a suitable zero-th order approximate solution with suitable error estimate. And the construction is given in subsection 4.1 in three steps.

Finally, the rest of the paper is organized as follows. We will first introduce some weighted Sobolev spaces and give some preliminaries in Section 2. The well-posedness of the linearized compressible Prandtl equations is given in Section 3. In Section 4, we introduce the Nash-MoserHömander iteration scheme, and construct the first approximate solution as the starting point of iteration. Then the local existence and uniqueness of solution to the nonlinear problem of the compressible Prandtl equations are proved.

\section{Preliminaries}

In this section, we will introduce some weighted Sobolev spaces and norms for later use. To simplify the notations, we denote by $\partial_{\tau}^{m}$ the summation of tangential derivatives $\partial_{\tau}^{m}=\partial_{t}^{m_{1}} \partial_{x}^{m_{2}}$ for all $m=\left(m_{1}, m_{2}\right) \in \mathbb{N}^{2},|m|=m_{1}+m_{2}$. Denote $\partial_{\tau}^{\alpha}$ by $Z_{1}^{\alpha},\left(\eta \partial_{\eta}\right)^{\alpha}$ by $Z_{2}^{\alpha}$ and $Z^{m}=Z_{1}^{m_{1}} Z_{2}^{m_{2}}$ for further simplification. Define

$$
\|f\|_{H_{c o, l}^{k_{1}, k_{2}}}=\left(\sum_{0 \leq m_{1} \leq k_{1}, 0 \leq m_{2} \leq k_{2}}\left\|\langle\eta\rangle^{l} Z_{1}^{m_{1}} Z_{2}^{m_{2}} f\right\|_{L^{2}\left([0, T] \times \mathbb{T} \times \mathbb{R}^{+}\right)}\right)^{1 / 2},
$$

and

$$
\begin{gathered}
\|f\|_{D_{c o, l}^{k_{1}, k_{2}}}=\left(\sum_{0 \leq m_{1} \leq k_{1}, 0 \leq m_{2} \leq k_{2}} \sup _{\eta \geq 0}\left\|\langle\eta\rangle^{l} Z_{1}^{m_{1}} Z_{2}^{m_{2}} f(\cdot, \eta)\right\|_{L^{2}([0, T] \times \mathbb{T})}\right)^{1 / 2}, \\
\|f\|_{C_{c o, l}^{k_{1}, k_{2}}}=\left(\sum_{0 \leq m_{1} \leq k_{1}, 0 \leq m_{2} \leq k_{2}} \sup _{(t, x) \in[0, T] \times \mathbb{T}}\left\|\langle\eta\rangle^{l} Z_{1}^{m_{1}} Z_{2}^{m_{2}} f(t, x, \cdot)\right\|_{L^{2}\left(\mathbb{R}^{+}\right)}\right)^{1 / 2} .
\end{gathered}
$$

Denote

$$
\|f\|_{H_{c o, l}^{k}}=\sum_{k_{1}+k_{2}=k}\|f\|_{H_{c o, l}^{k_{1}, k_{2}}} .
$$

The function spaces $D_{c o, l}^{k}$ and $C_{c o, l}^{k}$ can be defined similarly. Since the conormal operator $Z^{m}$ does not communicate with the normal derivative operator $\partial_{\eta}$, the following estimate of commutator is frequently used,

$$
\left\|\left[Z^{m}, \partial_{\eta}\right] f\right\|_{L_{l}^{2}} \lesssim\left\|\partial_{\eta} f\right\|_{H_{c o, l}^{m-1}} .
$$

Here and after $0<a \lesssim b$ means that there exists a uniform constant $C>0$ such that $a \leq C b$. The following weighted Sobolev spaces are also used frequently. Denote by $\partial_{\eta}^{k}$ the $k$-th normal derivative, for any given $k_{1}, k_{2} \in \mathbb{N}, l \in \mathbb{R}^{+}$and $0<T<+\infty$, set

$$
\|f\|_{\mathcal{B}_{l}^{k_{1}, k_{2}}}=\left(\sum_{0 \leq|m| \leq k_{1}, 0 \leq n \leq k_{2}}\left\|\langle\eta\rangle^{l} Z^{m} \partial_{\eta}^{n} f\right\|_{L^{2}\left([0, T] \times \mathbb{T} \times \mathbb{R}^{+}\right)}^{2}\right)^{1 / 2}
$$




$$
\begin{aligned}
& \|f\|_{\tilde{\mathcal{B}}_{l}^{k_{1}, k_{2}}}=\left(\sum_{0 \leq|m| \leq k_{1}, 0 \leq n \leq k_{2}}\left\|\langle\eta\rangle^{l} Z^{m} \partial_{\eta}^{n} f\right\|_{L^{\infty}\left([0, T] ; L^{2}\left(\mathbb{T} \times \mathbb{R}^{+}\right)\right)}^{2}\right)^{1 / 2}, \\
& \|f\|_{\mathcal{A}_{l}^{m}}=\left(\sum_{\left|m_{1}\right|+\left[\left(m_{2}+1\right) / 2\right] \leq|m|}\left\|\langle\eta\rangle^{l} Z^{m_{1}} \partial_{\eta}^{m_{2}} f\right\|_{L^{2}\left([0, T] \times \mathbb{T} \times \mathbb{R}^{+}\right)}^{2}\right)^{1 / 2} .
\end{aligned}
$$

It is straightforward to verify that

$$
\mathcal{A}_{l}^{m}=\bigcap_{m_{1}+\left[\left(m_{2}+1\right) / 2\right] \leq m} \mathcal{B}_{l}^{m_{1}, m_{2}} .
$$

We also define

$$
\|f\|_{\mathcal{D}_{l}^{m}}=\left(\sum_{k_{1}+\left[\left(k_{2}+1\right) / 2\right] \leq m}\left\|\langle\eta\rangle^{l} Z^{k_{1}} \partial_{\eta}^{k_{2}} f\right\|_{L_{\eta}^{\infty}\left(L_{t, x}^{2}\right)}^{2}\right)^{1 / 2}
$$

and

$$
\|f\|_{\mathcal{C}_{l}^{m}}=\left(\sum_{k_{1}+\left[\left(k_{2}+1\right) / 2\right] \leq m}\left\|\langle\eta\rangle^{l} Z^{k_{1}} \partial_{\eta}^{k_{2}} f\right\|_{L_{t, x}^{\infty}\left(L_{\eta}^{2}\right)}^{2}\right)^{1 / 2} .
$$

In addition, the homogeneous norms $\|\cdot\|_{\dot{\mathcal{A}}_{l}^{m}},\|\cdot\|_{\dot{\mathcal{C}}_{l}^{m}},\|\cdot\|_{\dot{\mathcal{D}}_{l}^{m}}$ correspond to the summation over $1 \leq\left|m_{1}\right|+\left[\left(m_{2}+1\right) / 2\right] \leq|m|$.

For $1 \leq p \leq+\infty$, we will use $\|f\|_{L_{l}^{p}\left(\mathbb{T} \times \mathbb{R}^{+}\right)}=\left\|\langle\eta\rangle^{l} f\right\|_{L^{p}\left(\mathbb{T} \times \mathbb{R}^{+}\right)}$. It is direct to show the following Sobolev type embeddings,

$$
\|f\|_{\mathcal{C}_{l}^{m}} \leq C_{s}\|f\|_{\mathcal{A}_{l}^{m+2}}, \quad\|f\|_{\mathcal{D}_{l}^{m}} \leq C_{s}\|f\|_{\mathcal{A}_{l+1}^{m+1}}
$$

Moreover, for any $l \geq 0$ and $m \geq 2$, the space $\mathcal{A}_{l}^{m}$ is continuously embedded into $\mathcal{C}_{b}^{m-2}$ which is the space of $(m-2)$-th order continuously differentiable functions with bounded derivatives. And the following Morse-type inequalities hold.

Lemma 2.1 For any proper functions $f$ and $g$, we have

$$
\|f g\|_{\mathcal{A}_{l}^{m}} \leq C_{m}\left\{\|f\|_{\mathcal{A}_{l}^{m}}\|g\|_{L^{\infty}}+\|f\|_{L^{\infty}}\|g\|_{\mathcal{\mathcal { A }}_{l}^{m}}\right\},
$$

and

$$
\|f g\|_{\mathcal{A}_{l}^{m}} \leq C_{m}\left\{\|f\|_{\mathcal{C}_{l}^{m}}\|g\|_{\mathcal{D}_{0}^{0}}+\|f\|_{\mathcal{C}_{l}^{0}}\|g\|_{\mathcal{D}_{0}^{m}}\right\} .
$$

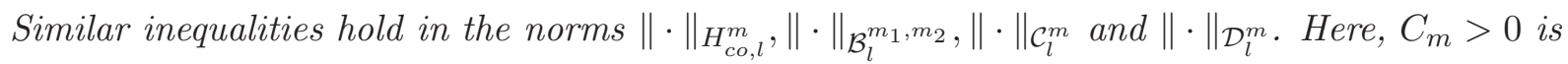
a constant depending only on $m$.

These results can be obtained similarly as those given in [10]. 


\section{Well-posedness of linearized system}

The strategy to prove the main result, Theorem 1.1, is to apply an iteration scheme to construct a sequence of approximate solution sequences, and then to show these approximate solutions converge in some suitable weighted Sobolev space. Since there is a loss of regularity, the NashMoser-Hömander iteration scheme is used for this purpose. In this section, we study the wellposedness of the linearized equations and obtain the required energy estimates of solutions to the linearized equations for the Nash-Moser-Hömander iteration.

Let $(\tilde{u}, \tilde{v})$ be a smooth background state satisfying the following conditions.

$$
\partial_{\eta} \tilde{u}(t, x, \eta)>0, \quad \partial_{x}(\bar{\rho} \tilde{u})+\partial_{\eta}(\bar{\rho} \tilde{v})=-\bar{\rho}_{t},\left.\quad \tilde{u}\right|_{\eta=0}=\left.\tilde{v}\right|_{\eta=0}=0, \quad \lim _{\eta \rightarrow+\infty} \tilde{u}=U(t, x) .
$$

Here $\tilde{v}$ is given by

$$
\tilde{v}=V(t, x) \eta+\frac{1}{\bar{\rho}(t, x)} \int_{0}^{\eta} \partial_{x}(\bar{\rho}(t, x)(U(t, x)-\tilde{u})) d \tilde{\eta} \triangleq V(t, x) \eta+\bar{v} .
$$

It extracts the linear increasing part $V(t, x) \eta$ by introducing the new function $\bar{v}$. The linearized problem of (1.1)-(1.3) around $(\tilde{u}, \tilde{v})$ can be written as

$$
\left\{\begin{array}{l}
u_{t}+\tilde{u} u_{x}+\tilde{v} \partial_{\eta} u+u \tilde{u}_{x}+\tilde{u}_{\eta} v-\frac{1}{\bar{\rho}} u_{\eta \eta}=f \\
\partial_{\eta}(\bar{\rho} v)+\partial_{x}(\bar{\rho} u)=0, \\
\left.u\right|_{\eta=0}=\left.v\right|_{\eta=0}=0, \quad \lim _{\eta \rightarrow+\infty} u(t, x, \eta)=0 \\
\left.u\right|_{t=0}=0
\end{array}\right.
$$

Similar to [1], by introducing the transformation

$$
\omega(t, x, \eta)=\left(\frac{\bar{\rho} u}{\partial_{\eta} \tilde{u}}\right)_{\eta}(t, x, \eta)
$$

then for classical solutions, from (3.1) we know that $w$ satisfies the following problem in $\{t>$ $0, x \in \mathbb{T}, \eta>0\}:$

$$
\left\{\begin{array}{l}
\omega_{t}+(\tilde{u} \omega)_{x}+(\eta V \omega)_{\eta}+(\bar{v} \omega)_{\eta}-\frac{2}{\bar{\rho}}\left(\omega \frac{\partial_{\eta}^{2} \tilde{u}}{\partial_{\eta} \tilde{u}}\right)_{\eta}+\left(\xi \int_{0}^{\eta} \omega(t, x, \tilde{\eta}) d \tilde{\eta}\right)_{\eta}-\frac{\bar{\rho} t}{\bar{\rho}} \omega-\frac{1}{\bar{\rho}} \omega_{\eta \eta}=\tilde{f}_{\eta} \\
\left.\frac{1}{\bar{\rho}}\left(\omega_{\eta}+2 \omega \frac{\partial_{\eta}^{2} \tilde{u}}{\partial_{\eta} \tilde{u}}\right)\right|_{\eta=0}=-\left.\tilde{f}\right|_{\eta=0} \\
\left.\omega\right|_{t=0}=0
\end{array}\right.
$$

where

$$
\xi=\frac{\left(\partial_{t}+\tilde{u} \partial_{x}+\tilde{v} \partial_{\eta}-\frac{1}{\bar{\rho}} \partial_{\eta}^{2}\right) \tilde{u}_{\eta}}{\tilde{u}_{\eta}}-\frac{\tilde{u} \bar{\rho}_{x}}{\bar{\rho}} \triangleq \xi_{1}-\frac{\tilde{u} \bar{\rho}_{x}}{\bar{\rho}}, \quad \tilde{f}=\frac{\bar{\rho} f}{\tilde{u}_{\eta}}
$$


To simplify the presentation, we use the notations:

$$
\lambda_{k_{1}, k_{2}}=\|\tilde{u}-U\|_{\mathcal{B}_{l}^{k_{1}, k_{2}}}+\left\|Z^{k_{1}} \partial_{\eta}^{k_{2}} \bar{v}\right\|_{L_{\eta}^{\infty}\left(L_{t, x}^{2}\right)}+\left\|Z^{k_{1}} \partial_{\eta}^{k_{2}} \chi\right\|_{L_{\eta}^{\infty}\left(L_{t, x}^{2}\right)}+\left\|\xi_{1}\right\|_{\mathcal{B}_{l}^{k_{1}, k_{2}}}
$$

with $\chi=\frac{\partial_{\eta}^{2} \tilde{u}}{\partial_{\eta} \tilde{u}}$. Set

$$
\lambda_{k}=\sum_{k_{1}+\left[\left(k_{2}+1\right) / 2\right] \leq k} \lambda_{k_{1}, k_{2}}
$$

Similar to [1], we have the following energy estimates of the solution to the problem (3.2).

Theorem 3.1 Suppose that the outer Euler flow $(\bar{\rho}(t, x), U(t, x), V(t, x)) \in H^{s}\left(\mathbb{R}_{+}^{2}\right)$, for $s$ suitably large, and $\bar{\rho}(t, x)$ has uniform lower positive bound. Moreover, for a given positive $k$, the compatibility condition for the problem (3.2) holds up to order $k$. Then for any fixed $l>1 / 2$, we have

$$
\|\omega\|_{\mathcal{A}_{l}^{k}} \leq C_{1}\left(\lambda_{4}\right)\|\tilde{f}\|_{\mathcal{A}_{l}^{k}}+C_{2}\left(\lambda_{4}\right) \lambda_{k}\|\tilde{f}\|_{\mathcal{A}_{l}^{3}}
$$

with $C_{1}(\cdot)$ and $C_{2}(\cdot)$ being two smooth functions in their arguments.

As we mentioned in the introduction, the main difference of the linear problem (3.2) from the one studied in [1] is that there is a linear growth term $\eta V$ in the equation of (3.2). Hence, we can not obtain the estimates of tangential derivatives directly as in [1. Similar to [12, we will study the linearized problem (3.2) in some conormal space. First, we have

Lemma 3.1 (L $L^{2}$-estimate) Under the assumptions in Theorem 3.1, there exists a positive constant $C$ such that

$$
\frac{d}{d t}\|\omega\|_{L_{l}^{2}\left(\mathbb{T} \times \mathbb{R}^{+}\right)}^{2}+\left\|\omega_{\eta}\right\|_{L_{l}^{2}\left(\mathbb{T} \times \mathbb{R}^{+}\right)}^{2} \leq C\left(\lambda_{3,1}+1\right)\|\omega\|_{L_{l}^{2}\left(\mathbb{T} \times \mathbb{R}^{+}\right)}^{2}+C\|\tilde{f}\|_{L_{l}^{2}\left(\mathbb{T} \times \mathbb{R}^{+}\right)}^{2} .
$$

Proof. Multiplying (3.2) by $\langle\eta\rangle^{2 l} \omega$ and integrating it over $\mathbb{T} \times \mathbb{R}^{+}$, we obtain

$$
\begin{aligned}
\frac{d}{d t}\|\omega\|_{L_{l}^{2}\left(\mathbb{T} \times \mathbb{R}^{+}\right)}^{2} & +2 \int_{\mathbb{T} \times \mathbb{R}^{+}}\langle\eta\rangle^{2 l} \omega\left\{(\tilde{u} \omega)_{x}+(\eta V \omega)_{\eta}+(\bar{v} \omega)_{\eta}-\frac{2}{\bar{\rho}}\left(\omega \frac{\partial_{\eta}^{2} \tilde{u}}{\partial_{\eta} \tilde{u}}\right)_{\eta}\right. \\
& \left.+\left(\xi \int_{0}^{\eta} \omega(t, x, \tilde{\eta}) d \tilde{\eta}\right)_{\eta}-\frac{\bar{\rho}_{t}}{\bar{\rho}} \omega-\frac{1}{\bar{\rho}} \omega_{\eta \eta}-\tilde{f}_{\eta}\right\} d x d \eta=0 .
\end{aligned}
$$

It is straightforward to obtain

$$
\begin{aligned}
& \int_{\mathbb{T} \times \mathbb{R}^{+}}\left\{(\tilde{u} \omega)_{x}+(\bar{v} \omega)_{\eta}\right\}\langle\eta\rangle^{2 l} \omega d x d \eta=\int_{\mathbb{T} \times \mathbb{R}^{+}}\langle\eta\rangle^{2 l} \frac{\omega^{2}}{2}\left(\frac{-\bar{\rho}_{t}-\bar{\rho} V-\tilde{u} \bar{\rho}_{x}}{\bar{\rho}}\right)-l \bar{v} \omega^{2}\langle\eta\rangle^{2 l-1} d x d \eta \\
& \lesssim\left(1+\|\tilde{u}\|_{L^{\infty}\left(\mathbb{T} \times \mathbb{R}^{+}\right)}+\|\bar{v}\|_{L^{\infty}\left(\mathbb{T} \times \mathbb{R}^{+}\right)}\right)\|\omega\|_{L_{l}^{2}\left(\mathbb{T} \times \mathbb{R}^{+}\right)}^{2},
\end{aligned}
$$


and

$$
\int_{\mathbb{T} \times \mathbb{R}^{+}}(\eta V \omega)_{\eta}\langle\eta\rangle^{2 l} \omega d x d \eta=\int_{\mathbb{T} \times \mathbb{R}^{+}} V \omega^{2}\left(\frac{1}{2}\langle\eta\rangle^{2 l}-l \eta\langle\eta\rangle^{2 l-1}\right) d x d \eta \lesssim\|\omega\|_{L_{l}^{2}\left(\mathbb{T} \times \mathbb{R}^{+}\right)}^{2} .
$$
we get

On the other hand, by integration by parts and using the boundary condition given in (3.2)

$$
\int_{\mathbb{T} \times \mathbb{R}^{+}}\left\{-\frac{2}{\bar{\rho}}(\omega \chi)_{\eta}-\frac{1}{\bar{\rho}} \omega_{\eta \eta}-\tilde{f}_{\eta}\right\}\langle\eta\rangle^{2 l} \omega d x d \eta=\int_{\mathbb{T} \times \mathbb{R}^{+}}\left\{\frac{2}{\bar{\rho}}(\omega \chi)+\frac{1}{\bar{\rho}} \omega_{\eta}+\tilde{f}\right\}\left(\langle\eta\rangle^{2 l} \omega\right)_{\eta} d x d \eta,
$$

where the right hand side can be estimated as follows.

$$
\begin{gathered}
\int_{\mathbb{T} \times \mathbb{R}^{+}} \frac{2}{\bar{\rho}} \omega \chi\left(\langle\eta\rangle^{2 l} \omega\right)_{\eta} d x d \eta \lesssim\|\chi\|_{L^{\infty}\left(\mathbb{T} \times \mathbb{R}^{+}\right)}\left(\|\omega\|_{L_{l}^{2}\left(\mathbb{T} \times \mathbb{R}^{+}\right)}^{2}+\|\omega\|_{L_{l}^{2}\left(\mathbb{T} \times \mathbb{R}^{+}\right)}\left\|\partial_{\eta} \omega\right\|_{L_{l}^{2}\left(\mathbb{T} \times \mathbb{R}^{+}\right)}\right), \\
\int_{\mathbb{T} \times \mathbb{R}^{+}} \frac{1}{\bar{\rho}} \omega_{\eta}\left(2 l\langle\eta\rangle^{2 l-1} \omega+\langle\eta\rangle^{2 l} \omega_{\eta}\right) \gtrsim\left\|\partial_{\eta} \omega\right\|_{L_{l}^{2}\left(\mathbb{T} \times \mathbb{R}^{+}\right)}^{2}-\|\omega\|_{L_{l}^{2}\left(\mathbb{T} \times \mathbb{R}^{+}\right)}^{2},
\end{gathered}
$$

and

$$
\int_{\mathbb{T} \times \mathbb{R}^{+}} \tilde{f}\left(2 l\langle\eta\rangle^{2 l-1} \omega+\langle\eta\rangle^{2 l} \omega_{\eta}\right) \lesssim\|\tilde{f}\|_{L_{l}^{2}\left(\mathbb{T} \times \mathbb{R}^{+}\right)}\left(\|\omega\|_{L_{l}^{2}\left(\mathbb{T} \times \mathbb{R}^{+}\right)}+\left\|\partial_{\eta} \omega\right\|_{L_{l}^{2}\left(\mathbb{T} \times \mathbb{R}^{+}\right)}\right) .
$$

Denote by

$$
\int_{\mathbb{T} \times \mathbb{R}^{+}}\left(\left(\xi_{1}-\frac{\tilde{u} \bar{\rho}_{x}}{\bar{\rho}}\right) \int_{0}^{\eta} \omega(t, x, \tilde{\eta}) d \tilde{\eta}\right)_{\eta}\langle\eta\rangle^{2 l} \omega d x d \eta \triangleq H^{1}+H^{2} .
$$

As $l>1 / 2$, by integration by parts, it follows

$$
\left|H^{1}\right| \lesssim\left\|\xi_{1}\right\|_{L_{x}^{\infty}\left(L_{\eta, l}^{2}\right)}\left(\|\omega\|_{L_{l}^{2}\left(\mathbb{T} \times \mathbb{R}^{+}\right)}^{2}+\|\omega\|_{L_{l}^{2}\left(\mathbb{T} \times \mathbb{R}^{+}\right)}\left\|\partial_{\eta} \omega\right\|_{L_{l}^{2}\left(\mathbb{T} \times \mathbb{R}^{+}\right)}\right),
$$

and

$$
\begin{aligned}
\left|H^{2}\right| \leq & \left|\int_{\mathbb{T} \times \mathbb{R}^{+}} \frac{\bar{\rho}_{x}(\tilde{u}-U)}{\bar{\rho}} \int_{0}^{\eta} \omega(t, x, \tilde{\eta}) d \tilde{\eta}\left(\langle\eta\rangle^{2 l} \omega\right)_{\eta} d x d \eta\right| \\
& +\left|\int_{\mathbb{T} \times \mathbb{R}^{+}}\left(\frac{\bar{\rho}_{x} U}{\bar{\rho}} \int_{0}^{\eta} \omega(t, x, \tilde{\eta}) d \tilde{\eta}\right)_{\eta}\langle\eta\rangle^{2} \omega d x d \eta\right| \\
& \lesssim\|\omega\|_{L_{l}^{2}\left(\mathbb{T} \times \mathbb{R}^{+}\right)}^{2}+\|\tilde{u}-U\|_{L_{x}^{\infty}\left(L_{\eta, l}^{2}\right)}\left(\|\omega\|_{L_{l}^{2}\left(\mathbb{T} \times \mathbb{R}^{+}\right)}^{2}+\|\omega\|_{L_{l}^{2}\left(\mathbb{T} \times \mathbb{R}^{+}\right)}\left\|\partial_{\eta} \omega\right\|_{L_{l}^{2}\left(\mathbb{T} \times \mathbb{R}^{+}\right)}\right) .
\end{aligned}
$$

Thus, from (3.7) we obtain

$$
\frac{d}{d t}\|\omega\|_{L_{l}^{2}\left(\mathbb{T} \times \mathbb{R}^{+}\right)}^{2}+\left\|\omega_{\eta}\right\|_{L_{l}^{2}\left(\mathbb{T} \times \mathbb{R}^{+}\right)}^{2} \leq C\left(1+\lambda_{3,1}\right)\|\omega\|_{L_{l}^{2}\left(\mathbb{T} \times \mathbb{R}^{+}\right)}^{2}+C\|\tilde{f}\|_{L_{l}^{2}\left(\mathbb{T} \times \mathbb{R}^{+}\right)}^{2},
$$

by noting

$$
\|\tilde{u}, \bar{v}, \chi\|_{L^{\infty}\left(\mathbb{T} \times \mathbb{R}^{+}\right)}+\left\|\xi_{1}\right\|_{L_{x}^{\infty}\left(L_{\eta, l}^{2}\right)}+\|\tilde{u}-U\|_{L_{x}^{\infty}\left(L_{\eta, l}^{2}\right)} \lesssim\left(1+\lambda_{3,1}\right) .
$$

It completes the proof of the estimate (3.6). 
Lemma 3.2 (Estimates of conormal derivatives) Under the assumptions in Theorem 3.1, for any fixed $T>0$, there exists a positive constant $C>0$ such that

$$
\begin{aligned}
& \frac{d}{d t}\|\omega\|_{H_{c o, l}^{m}}^{2}+\left\|\omega_{\eta}\right\|_{H_{c o, l}^{m}}^{2} \\
\leq & C\left(1+\lambda_{3,1}^{2}\right)\|\omega\|_{H_{c o, l}^{m}}^{2}+C\left(\|\tilde{f}\|_{H_{c o, l}^{m}}^{2}+\left\|\tilde{f}_{\eta}\right\|_{H_{c o, l}^{m-1}}^{2}+\left(\lambda_{m-1,1}^{2}+\lambda_{m, 0}^{2}+1\right)\|\omega\|_{\mathcal{B}_{l}^{3,1}}^{2}\right)
\end{aligned}
$$

holds for $t \in[0, T]$.

Proof. The proof is divided into four steps.

(1) Applying the conormal derivative operator $Z^{m}$ on the equation in (3.2), multiplying the resulting equation by $\langle\eta\rangle^{2 l} Z^{m} \omega$ and integrating it over $\mathbb{T} \times \mathbb{R}^{+}$, it follows

$$
\begin{aligned}
\frac{d}{d t}\left\|Z^{m} \omega\right\|_{L_{l}^{2}\left(\mathbb{T} \times \mathbb{R}^{+}\right)}^{2} & +2 \int_{\mathbb{T} \times \mathbb{R}^{+}}\langle\eta\rangle^{2 l} Z^{m} \omega Z^{m}\left\{(\tilde{u} \omega)_{x}+(\eta V \omega)_{\eta}+(\bar{v} \omega)_{\eta}-\frac{2}{\bar{\rho}}\left(\omega \frac{\partial_{\eta}^{2} \tilde{u}}{\partial_{\eta} \tilde{u}}\right)_{\eta}\right. \\
& \left.+\left(\xi \int_{0}^{\eta} \omega(t, x, \tilde{\eta}) d \tilde{\eta}\right)_{\eta}-\frac{\bar{\rho}_{t}}{\bar{\rho}} \omega-\frac{1}{\bar{\rho}} \omega_{\eta \eta}-\tilde{f}_{\eta}\right\} d x d \eta=0 .
\end{aligned}
$$

Now, let us estimate each term of (3.9). Denote

$$
\left\{\begin{array}{l}
I_{1}=\int_{\mathbb{T} \times \mathbb{R}^{+}} Z^{m}\left[(\tilde{u} \omega)_{x}+(\bar{v} \omega)_{\eta}\right]\langle\eta\rangle^{2 l} Z^{m} \omega d x d \eta \\
I_{2}=-\int_{\mathbb{T} \times \mathbb{R}^{+}}\langle\eta\rangle^{2 l} Z^{m} \omega Z^{m}\left(\frac{2}{\bar{\rho}}\left(\omega \frac{\partial_{\eta}^{2} \tilde{u}}{\partial_{\eta} \tilde{u}}\right)_{\eta}+\frac{1}{\bar{\rho}} \omega_{\eta \eta}+\tilde{f}_{\eta}\right) d x d \eta \\
I_{3}=\int_{\mathbb{T} \times \mathbb{R}^{+}}\langle\eta\rangle^{2 l} Z^{m} \omega Z^{m}\left(\xi \int_{0}^{\eta} \omega(t, x, \tilde{\eta}) d \tilde{\eta}\right)_{\eta} d x d \eta
\end{array}\right.
$$

(2) Estimate of $I_{1}$. Obviously, we have

$$
\begin{aligned}
I_{1} & =\int_{\mathbb{T} \times \mathbb{R}^{+}} Z^{m}\left[(\tilde{u} \omega)_{x}+(\bar{v} \omega)_{\eta}\right]\langle\eta\rangle^{2 l} Z^{m} \omega d x d \eta \\
& =\int_{\mathbb{T} \times \mathbb{R}^{+}} Z^{m}\left[\left(-\frac{\bar{\rho}_{t}+\bar{\rho} V}{\bar{\rho}}\right) \omega+\left(-\frac{\bar{\rho}_{x} \tilde{u}}{\bar{\rho}}\right) \omega+\tilde{u} \omega_{x}+\bar{v} \omega_{\eta}\right]\langle\eta\rangle^{2 l} Z^{m} \omega d x d \eta \\
& \triangleq I_{1}^{1}+I_{1}^{2}+I_{1}^{3}+I_{1}^{4},
\end{aligned}
$$

and

$$
\begin{aligned}
\left|I_{1}^{1}\right| & =\left|\sum_{m_{1}+m_{2}=m} C_{m}^{m_{1}} \int_{\mathbb{T} \times \mathbb{R}^{+}}\langle\eta\rangle^{2 l}\left(Z^{m_{1}}\left(-\frac{\bar{\rho}_{t}+\bar{\rho} V}{\bar{\rho}}\right)\right)\left(Z^{m_{2}} \omega\right)\left(Z^{m} \omega\right) d x d \tau\right| \lesssim\|\omega\|_{H_{c o, l}^{m},}^{2}, \\
\left|I_{1}^{2}\right| & =\left|\sum_{m_{1}+m_{2}=m} C_{m}^{m_{1}} \int_{\mathbb{T} \times \mathbb{R}^{+}} Z^{m_{1}}\left[\left(\frac{\bar{\rho}_{x}(\tilde{u}-U(x, t))}{\bar{\rho}}\right)+\frac{\bar{\rho}_{x} U}{\bar{\rho}}\right] Z^{m_{2}} \omega\langle\eta\rangle^{2 l} Z^{m} \omega d x d \eta\right|
\end{aligned}
$$




$$
\lesssim\|\tilde{u}-U\|_{H_{c o, l}^{m}}\|\omega\|_{L^{\infty}}\|\omega\|_{H_{c o, l}^{m}}+\left(1+\|\tilde{u}\|_{L^{\infty}}\right)\|\omega\|_{H_{c o, l}^{m}}^{2} .
$$

On the other hand, one has

$$
\begin{aligned}
I_{1}^{3}= & \sum_{m_{1}+m_{2}=m, m_{2}<m} C_{m}^{m_{1}} \int_{\mathbb{T} \times \mathbb{R}^{+}}\langle\eta\rangle^{2 l}\left(Z^{m_{1}} \tilde{u}\right)\left(Z^{m_{2}} \omega_{x}\right)\left(Z^{m} \omega\right) d x d \eta \\
& +\int_{\mathbb{T} \times \mathbb{R}^{+}}\langle\eta\rangle^{2 l} \tilde{u}\left(Z^{m} \omega_{x}\right)\left(Z^{m} \omega\right) d x d \eta \triangleq I_{1}^{3 a}+I_{1}^{3 b},
\end{aligned}
$$

and

$$
\begin{aligned}
I_{1}^{4}= & \sum_{m_{1}+m_{2}=m, m_{2}<m} C_{m}^{m_{1}} \int_{\mathbb{T} \times \mathbb{R}^{+}}\langle\eta\rangle^{2 l}\left(Z^{m_{1}} \bar{v}\right)\left(Z^{m_{2}} \omega_{\eta}\right)\left(Z^{m} \omega\right) d x d \eta \\
& +\int_{\mathbb{T} \times \mathbb{R}^{+}}\langle\eta\rangle^{2 l} \bar{v}\left(Z^{m} \omega_{\eta}\right)\left(Z^{m} \omega\right) d x d \eta \triangleq I_{1}^{4 a}+I_{1}^{4 b} .
\end{aligned}
$$

Note that $I_{1}^{3 a}$ can be estimated similarly as $I_{1}^{2}$, and

$$
\left|I_{1}^{4 a}\right| \lesssim\left\|Z^{m} \omega\right\|_{L_{l}^{2}}\left(\left\|Z^{m} \bar{v}\right\|_{L_{\eta}^{\infty}\left(L_{x}^{2}\right)}\left\|\omega_{\eta}\right\|_{L_{\eta}^{2}\left(L_{x}^{\infty}\right)}+\|Z \bar{v}\|_{L^{\infty}}\left\|Z^{m-1} \omega_{\eta}\right\|_{L_{l}^{2}}\right) .
$$

By using $\partial_{\eta}(\bar{\rho} \bar{v})+\partial_{x}(\bar{\rho} \tilde{u})=-\bar{\rho}_{t}-\bar{\rho} V$, integration by parts and the commutator estimate (2.1), we obtain

$$
\left|I_{1}^{3 b}+I_{1}^{4 b}\right| \lesssim\left(1+\|\tilde{u}\|_{L^{\infty}}+\|\bar{v}\|_{\left.L^{\infty}\right)}\left\|Z^{m} \omega\right\|_{L_{l}^{2}\left(\mathbb{T} \times \mathbb{R}^{+}\right)}^{2}+\|\bar{v}\|_{L^{\infty}}\left\|\partial_{\eta} \omega\right\|_{H_{c o, l}^{m-1}}\left\|Z^{m} \omega\right\|_{L_{l}^{2}\left(\mathbb{T} \times \mathbb{R}^{+}\right)} .\right.
$$

Moreover, the definition of the operator $Z_{2}$ gives that

$$
\int_{\mathbb{T} \times \mathbb{R}^{+}} Z^{m}(\eta V \omega)_{\eta}\langle\eta\rangle^{2 l} Z^{m} \omega d x d \eta=\int_{\mathbb{T} \times \mathbb{R}^{+}} Z^{m}\left(V \omega+V \eta \omega_{\eta}\right)\langle\eta\rangle^{2 l} Z^{m} \omega d x d \eta \lesssim\|\omega\|_{H_{c o, l}^{m}}^{2} .
$$

(3) Estimate of $I_{2}$. First, by using the boundary condition given in (3.2), we have

$$
\begin{aligned}
I_{2}= & \int_{\mathbb{T} \times \mathbb{R}^{+}}\left(\langle\eta\rangle^{2 l} Z^{m} \omega\right)_{\eta} Z^{m}\left(\frac{2}{\bar{\rho}} \omega \frac{\partial_{\eta}^{2} \tilde{u}}{\partial_{\eta} \tilde{u}}+\frac{1}{\bar{\rho}} \omega_{\eta}+\tilde{f}\right) d x d \eta \\
& -\int_{{\mathbb{T} \times \mathbb{R}^{+}}}\langle\eta\rangle^{2 l} Z^{m} \omega\left[Z^{m}, \partial_{\eta}\right]\left(\frac{2}{\bar{\rho}} \omega \frac{\partial_{\eta}^{2} \tilde{u}}{\partial_{\eta} \tilde{u}}+\frac{1}{\bar{\rho}} \omega_{\eta}+\tilde{f}\right) d x d \eta \\
\triangleq & I_{2}^{1}+I_{2}^{2}+I_{2}^{3}+I_{2}^{4},
\end{aligned}
$$

with $I_{2}^{4}$ being the terms involving $\tilde{f}$,

$$
\begin{gathered}
I_{2}^{1}=\int_{\mathbb{T} \times \mathbb{R}^{+}}\left(\langle\eta\rangle^{2 l} Z^{m} \omega\right)_{\eta} Z^{m}\left(\frac{2}{\bar{\rho}} \omega \frac{\partial_{\eta}^{2} \tilde{u}}{\partial_{\eta} \tilde{u}}\right) d x d \eta-\int_{\mathbb{T} \times \mathbb{R}^{+}}\langle\eta\rangle^{2 l} Z^{m} \omega\left[Z^{m}, \partial_{\eta}\right]\left(\frac{2}{\bar{\rho}} \omega \frac{\partial_{\eta}^{2} \tilde{u}}{\partial_{\eta} \tilde{u}}\right) d x d \eta, \\
I_{2}^{2}=\int_{\mathbb{T} \times \mathbb{R}^{+}}\left(\langle\eta\rangle^{2 l} Z^{m} \omega\right)_{\eta} Z^{m}\left(\frac{1}{\bar{\rho}} \omega_{\eta}\right) d x d \eta,
\end{gathered}
$$


and

$$
I_{2}^{3}=-\int_{\mathbb{T} \times \mathbb{R}^{+}}\langle\eta\rangle^{2 l} Z^{m} \omega\left[Z^{m}, \partial_{\eta}\right]\left(\frac{1}{\bar{\rho}} \omega_{\eta}\right) d x d \eta .
$$

It is straightforward to show that

$$
\begin{aligned}
& \left|I_{2}^{1}\right| \leq\left|\int_{\mathbb{T} \times \mathbb{R}^{+}} Z^{m}\left(\frac{2}{\bar{\rho}} \chi \omega\right)\langle\eta\rangle^{2 l}\left(Z^{m} \omega_{\eta}+\frac{2 l}{\langle\eta\rangle} Z^{m} \omega+\left[Z^{m}, \partial_{\eta}\right] \omega\right) d x d \eta\right|+\left\|Z^{m} \omega\right\|_{L_{l}^{2}}\left\|\frac{2}{\bar{\rho}}(\chi \omega)_{\eta}\right\|_{H_{c o, l}^{m-1}} \\
& \lesssim\left\|\frac{2}{\bar{\rho}} \chi \omega\right\|_{H_{c o, l}^{m}}\left(\left\|Z^{m} \omega_{\eta}\right\|_{L_{l}^{2}}+\left\|Z^{m} \omega\right\|_{L_{l}^{2}}+\left\|\partial_{\eta} \omega\right\|_{H_{c o, l}^{m-1}}\right)+\left\|Z^{m} \omega\right\|_{L_{l}^{2}}\left\|\frac{2}{\bar{\rho}}(\chi \omega)_{\eta}\right\|_{H_{c o, l}^{m-1}} \\
& \lesssim\left(\|\chi\|_{l \infty}\|\omega\|_{H_{c o, l}^{m}}+\|\chi\|_{D_{c o, 0}^{m}}\|\omega\|_{L_{\eta, l}^{2}\left(L_{x}^{\infty}\right)}\right)\left(\|\omega\|_{H_{c o, l}^{m}}+\left\|\partial_{\eta} \omega\right\|_{H_{c o, l}^{m}}\right) \\
& +\left\|Z^{m} \omega\right\|_{L_{l}^{2}}\left(\left\|\partial_{\eta} \chi\right\|_{D_{c o, 0}^{m-1,0}}\|\omega\|_{L_{\eta, l}^{2}\left(L_{x}^{\infty}\right)}+\left\|\partial_{\eta} \chi\right\|_{L^{\infty}}\|\omega\|_{H_{c o, l}^{m-1}}+\|\chi\|_{D_{c o, 0}^{m-1,0}}\left\|\omega_{\eta}\right\|_{L_{\eta, l}^{2}\left(L_{x}^{\infty}\right)}+\|\chi\|_{L^{\infty}}\left\|\omega_{\eta}\right\|_{H_{c o, l}^{m-1}}\right), \\
& I_{2}^{2}=\int_{\mathbb{T} \times \mathbb{R}^{+}}\left(Z^{m} \frac{\omega_{\eta}}{\bar{\rho}}\right)\left(2 l\langle\eta\rangle^{2 l-1} Z^{m} \omega+\langle\eta\rangle^{2 l}\left(Z^{m} \omega\right)_{\eta}\right) d x d \eta \\
& \gtrsim \frac{1}{2}\left\|Z^{m} \omega_{\eta}\right\|_{L_{l}^{2}}^{2}-\|\omega\|_{H_{c o, l}^{m}}^{2}-\left\|\omega_{\eta}\right\|_{H_{c o, l}^{m-1}}^{2},
\end{aligned}
$$

and

$$
\left|I_{2}^{3}\right| \leq\left\|\partial_{\eta}\left(\frac{\omega_{\eta}}{\bar{\rho}}\right)\right\|_{H_{c o, l}^{m-1}}\left\|Z^{m} \omega\right\|_{L_{l}^{2}}
$$

From the equation (3.2), we have

$$
\begin{aligned}
& \left\|\partial_{\eta}\left(\frac{\omega_{\eta}}{\bar{\rho}}\right)\right\|_{H_{c o, l}^{m-1}} \\
= & \left\|\omega_{t}+(\tilde{u} \omega)_{x}+(\eta V \omega)_{\eta}+(\bar{v} \omega)_{\eta}-\frac{2}{\bar{\rho}}\left(\omega \frac{\partial_{\eta}^{2} \tilde{u}}{\partial_{\eta} \tilde{u}}\right)_{\eta}+\left(\xi \int_{0}^{\eta} \omega(t, x, \tilde{\eta}) d \tilde{\eta}\right)_{\eta}-\frac{\bar{\rho}_{t}}{\bar{\rho}} \omega-\tilde{f}_{\eta}\right\|_{H_{c o, l}^{m-1}} .
\end{aligned}
$$

The terms on the right hand side of the above equation can be estimated as

$$
\begin{gathered}
\left\|\omega_{t}+(\eta V \omega)_{\eta}-\frac{\bar{\rho}_{t}}{\rho} \omega\right\|_{H_{c o, l}^{m-1}} \lesssim\|\omega\|_{H_{c o, l}^{m},} \\
\left\|(\tilde{u} \omega)_{x}+(\bar{v} \omega)_{\eta}-\frac{2}{\bar{\rho}}\left(\omega \frac{\partial_{\eta}^{2} \tilde{u}}{\partial_{\eta} \tilde{u}}\right)_{\eta}\right\|_{H_{c o, l}^{m-1}} \\
=\left\|-\left(\frac{\bar{\rho}_{t}+\bar{\rho} V+\bar{\rho}_{x} \tilde{u}}{\bar{\rho}}+\frac{2}{\bar{\rho}}\left(\frac{\partial_{\eta}^{2} \tilde{u}}{\partial_{\eta} \tilde{u}}\right)_{\eta}\right) \omega+\tilde{u} \omega_{x}+\left(\bar{v}-\frac{2}{\bar{\rho}} \frac{\partial_{\eta}^{2} \tilde{u}}{\partial_{\eta} \tilde{u}}\right) \omega_{\eta}\right\|_{H_{c o, l}^{m-1}} \\
\leq\left(1+\|\tilde{u}\|_{L^{\infty}}+\left\|\chi_{\eta}\right\|_{L^{\infty}}\right)\|\omega\|_{H_{c o, l}^{m}}+\left(\|\bar{v}\|_{L^{\infty}}+\|\chi\|_{L^{\infty}}\right)\left\|\omega_{\eta}\right\|_{H_{c o, l}^{m-1}} \\
+\left(\|\tilde{u}-U\|_{H_{c o, l}^{m-1}}+\left\|\chi_{\eta}\right\|_{H_{c o, l}^{m-1}}\right)\left(\|\omega\|_{L^{\infty}}+\left\|\omega_{x}\right\|_{L^{\infty}}\right)+\left(\|\bar{v}\|_{D_{c o, l}^{m, 0}}+\|\chi\|_{\left.D_{c o, l}^{m, 0}\right)}\right)\left\|\omega_{\eta}\right\|_{L_{\eta, l}^{2}\left(L_{x}^{\infty}\right)},
\end{gathered}
$$


and

$$
\begin{aligned}
& \left\|\left(\xi \int_{0}^{\eta} \omega(t, x, \tilde{\eta}) d \tilde{\eta}\right)_{\eta}\right\|_{H_{c o, l}^{m-1}} \\
& \leq\left(\left\|\xi_{1}\right\|_{H_{c o, l}^{m-1}}+\|\tilde{u}-U\|_{H_{c o, l}^{m-1}}\right)\|\omega\|_{L^{\infty}}+\|\xi\|_{L^{\infty}}\|\omega\|_{H_{c o, l}^{m-1}} \\
& +\left\|\left(\xi_{1}\right)_{\eta},(\tilde{u}-U)_{\eta}\right\|_{H_{c o, l}^{m-1}}\|\omega\|_{L_{\eta, l}^{2}\left(L_{x}^{\infty}\right)}+\left\|\left(\xi_{1}\right)_{\eta},(\tilde{u}-U)_{\eta}\right\|_{L_{\eta, l}^{2}\left(L_{x}^{\infty}\right)}\|\omega\|_{H_{c o, l}^{m-1}}
\end{aligned}
$$

(4) Estimate of $I_{3}$. Decompose $I_{3}$ into

$$
I_{3}=I_{3}^{1}+I_{3}^{2},
$$

with

$$
I_{3}^{1}=\int_{\mathbb{T} \times \mathbb{R}^{+}}\langle\eta\rangle^{2 l} Z^{m} \omega Z^{m}\left(\xi_{1} \int_{0}^{\eta} \omega(t, x, \tilde{\eta}) d \tilde{\eta}\right)_{\eta} d x d \eta
$$

and

$$
I_{3}^{2}=-\int_{\mathbb{T} \times \mathbb{R}^{+}}\langle\eta\rangle^{2 l} Z^{m} \omega Z^{m}\left(\frac{\tilde{u} \bar{\rho}_{x}}{\bar{\rho}} \int_{0}^{\eta} \omega(t, x, \tilde{\eta}) d \tilde{\eta}\right)_{\eta} d x d \eta .
$$

For $l>1 / 2$, we have

$$
\begin{aligned}
\left|I_{3}^{1}\right| \leq & \left|\int_{\mathbb{T}^{\prime} \times \mathbb{R}^{+}} Z^{m}\left(\xi_{1} \int_{0}^{\eta} \omega d \tilde{\eta}\right)\left(\langle\eta\rangle^{2 l} Z^{m} \omega\right)_{\eta} d x d \eta\right|+\left|\int_{\mathbb{T} \times \mathbb{R}^{+}}\left[Z^{m}, \partial_{\eta}\right]\left(\xi_{1} \int_{0}^{\eta} \omega d \tilde{\eta}\right)\left(\langle\eta\rangle^{2 l} Z^{m} \omega\right) d x d \eta\right| \\
\lesssim & \left(\left\|\xi_{1}\right\|_{L_{\eta, l}^{2}\left(L_{x}^{\infty}\right)}\|\omega\|_{H_{c o, l}^{m}}+\left\|Z^{m} \xi_{1}\right\|_{L_{l}^{2}}\|\omega\|_{L_{\eta, l}^{2}\left(L_{x}^{\infty}\right)}\right)\left(\left\|Z^{m} \omega\right\|_{L_{l}^{2}}+\left\|\omega_{\eta}\right\|_{H_{c o, l}^{m}}\right) \\
& +\left\|Z^{m} \omega\right\|_{L_{l}^{2}}\left\|\partial_{\eta}\left(\xi_{1} \int_{0}^{\eta} \omega d \tilde{\eta}\right)\right\|_{H_{c o, l}^{m-1}} \\
\lesssim & \left(\left\|\xi_{1}\right\|_{L_{\eta, l}^{2}\left(L_{x}^{\infty}\right)}\|\omega\|_{H_{c o, l}^{m}}+\left\|Z^{m} \xi_{1}\right\|_{L_{l}^{2}}\|\omega\|_{L_{\eta, l}^{2}\left(L_{x}^{\infty}\right)}\right)\left(\left\|Z^{m} \omega\right\|_{L_{l}^{2}}+\left\|\omega_{\eta}\right\|_{H_{c o, l}^{m}}\right) \\
& +\left\|Z^{m} \omega\right\|_{L_{l}^{2}}\left(\left\|\xi_{1}\right\|_{H_{c o, l}^{m-1}}\|\omega\|_{L_{l}^{\infty}}+\left\|\xi_{1}\right\|_{L_{l}^{\infty}}\|\omega\|_{H_{c o, l}^{m-1}}\right. \\
& \left.+\left\|\left(\xi_{1}\right)_{\eta}\right\|_{H_{c o, l}^{m-1}}\|\omega\|_{L_{\eta, l}^{2}\left(L_{x}^{\infty}\right)}+\left\|\left(\xi_{1}\right)_{\eta}\right\|_{L_{\eta, l}^{2}\left(L_{x}^{\infty}\right)}\|\omega\|_{H_{c o, l}^{m-1}}\right),
\end{aligned}
$$

and

$$
\begin{aligned}
\left|I_{3}^{2}\right|= & \left|\int_{\mathbb{T} \times \mathbb{R}^{+}} Z^{m}\left(\frac{\bar{\rho}_{x}(\tilde{u}-U+U)}{\bar{\rho}} \int_{0}^{\eta} \omega(t, x, \tilde{\eta}) d \tilde{\eta}\right)_{\eta}\langle\eta\rangle^{2 l} Z^{m} \omega d x d \eta\right| \\
\lesssim & \left(\left\|Z^{m}(\tilde{u}-U)\right\|_{L_{l}^{2}\|\omega\|_{L_{\eta, l}^{2}\left(L_{x}^{\infty}\right)}}+\|\tilde{u}-U\|_{L_{\eta, l}^{2}\left(L_{x}^{\infty}\right)}\|\omega\|_{H_{c o, l}^{m}}\right)\left(\left\|Z^{m} \omega\right\|_{L_{l}^{2}}+\left\|\omega_{\eta}\right\|_{H_{c o, l}^{m}}\right) \\
& +\left\|Z^{m} \omega\right\|_{L_{l}^{2}}\left(\left\|\partial_{\eta}(\tilde{u}-U)\right\|_{L_{l}^{\infty}}\|\omega\|_{H_{c o, l}^{m-1}}+\left\|\partial_{\eta}(\tilde{u}-U)\right\|_{H_{c o, l}^{m-1}}\|\omega\|_{L_{\eta, l}^{2}\left(L_{x}^{\infty}\right)}\right. \\
& \left.+\|\tilde{u}-U\|_{H_{c o, l}^{m-1}}\|\omega\|_{L^{\infty}}+\|\tilde{u}-U\|_{L^{\infty}}\|\omega\|_{H_{c o, l}^{m-1}}+\left\|Z^{m} \omega\right\|_{L_{l}^{2}}\right) .
\end{aligned}
$$

Summarizing the above estimates, it follows

$$
\begin{aligned}
& \frac{d}{d t}\|\omega\|_{H_{c o, l}^{m}}^{2}+\left\|\omega_{\eta}\right\|_{H_{c o, l}^{m}}^{2} \\
\lesssim & \|\tilde{f}\|_{H_{c o, l}^{m}}^{m}+\left\|\tilde{f}_{\eta}\right\|_{H_{c o, l}^{m-1}}^{2}+\left(1+\lambda_{3,1}^{2}\right)\|\omega\|_{H_{c o, l}^{m}}^{2}+\left(\lambda_{m, 0}^{2}+\lambda_{m-1,1}^{2}+1\right)\|\omega\|_{\mathcal{B}_{l}^{3,1}}^{2},
\end{aligned}
$$


where we have used the inequalities

$$
\left\|\omega, \omega_{x}\right\|_{L_{l}^{\infty}\left(\mathbb{T} \times \mathbb{R}^{+}\right)}, \quad\left\|\omega, \omega_{\eta}\right\|_{L_{\eta, l}^{2}\left(L_{x}^{\infty}\right)} \leq C\|\omega\|_{\mathcal{B}_{l}^{3,1}} .
$$

And this completes the proof of the lemma.

Remark 3.1 Similar to the above proof, one can obtain

$$
\|\omega\|_{\mathcal{B}_{l}^{m, 1}}^{2} \leq C\left(\|\tilde{f}\|_{H_{c o, l}^{m}}^{2}+\left\|\tilde{f}_{\eta}\right\|_{H_{c o, l}^{m-1}}^{2}\right), \quad 0 \leq m \leq 3
$$

When $m=0$, the term $\left\|\tilde{f}_{\eta}\right\|_{H_{c o, l}^{m-1}}^{2}$ is not in (3.11). By combining (3.10), (3.11) and using Gronwall's inequality, we get

$$
\|\omega\|_{\mathcal{B}_{l}^{m, 1}}^{2} \leq C\left(\|\tilde{f}\|_{\mathcal{B}_{l}^{m, 0}}^{2}+\|\tilde{f}\|_{\mathcal{B}_{l}^{m-1,1}}^{2}\right)+\left(1+\lambda_{m-1,1}^{2}+\lambda_{m, 0}^{2}\right)\left(\|\tilde{f}\|_{\mathcal{B}_{l}^{3,0}}^{2}+\|\tilde{f}\|_{\mathcal{B}_{l}^{2,1}}^{2}\right) .
$$

We are now ready to prove Theorem 3.1 .

Proof of Theorem 3.1; It remains to estimate the higher order normal derivatives.

From the equation given in (3.2), we have

$$
\frac{1}{\bar{\rho}} \omega_{\eta \eta}=\omega_{t}+(\tilde{u} \omega)_{x}+(\eta V \omega)_{\eta}+(\bar{v} \omega)_{\eta}-\frac{2}{\bar{\rho}}\left(\omega \frac{\partial_{\eta}^{2} \tilde{u}}{\partial_{\eta} \tilde{u}}\right)_{\eta}+\left(\xi \int_{0}^{\eta} \omega(t, x, \tilde{\eta}) d \tilde{\eta}\right)_{\eta}-\frac{\bar{\rho}_{t}}{\bar{\rho}} \omega-\tilde{f}_{\eta} .
$$

Applying the conormal operator $Z^{m}$ to the above equation (3.13) gives

$$
\begin{aligned}
\|\omega\|_{\mathcal{B}_{l}^{m, 2}} \leq & \left\|\bar{\rho} \omega_{t}\right\|_{\mathcal{B}_{l}^{m, 0}}+\left\|\bar{\rho}\left((\tilde{u} \omega)_{x}+(\bar{v} \omega)_{\eta}\right)\right\|_{\mathcal{B}_{l}^{m, 0}}+\left\|\bar{\rho}(\eta V \omega)_{\eta}\right\|_{\mathcal{B}_{l}^{m, 0}}+\|2 \chi \omega\|_{\mathcal{B}_{l}^{m, 1}} \\
& +\left\|\bar{\rho} \partial_{\eta}\left(\xi \int_{0}^{\eta} \omega(t, x, \tilde{\eta}) d \tilde{\eta}\right)\right\|_{\mathcal{B}_{l}^{m, 0}}+\left\|\bar{\rho}_{t} \omega\right\|_{\mathcal{B}_{l}^{m, 0}}+\left\|\bar{\rho} \partial_{\eta} \tilde{f}\right\|_{\mathcal{B}_{l}^{m, 0}}
\end{aligned}
$$

We estimate each term on the right hand side of the above inequality. By using Lemma 2.1, we get

$$
\left\|\bar{\rho} \omega_{t}\right\|_{\mathcal{B}_{l}^{m, 0}}+\left\|(\eta V \omega)_{\eta}\right\|_{\mathcal{B}_{l}^{m, 0}}+\left\|\bar{\rho}_{t} \omega\right\|_{\mathcal{B}_{l}^{m, 0}} \lesssim\|\omega\|_{\mathcal{B}_{l}^{m+1,0}}
$$

Obviously, it holds

$$
\left\|\bar{\rho}\left((\tilde{u} \omega)_{x}+(\bar{v} \omega)_{\eta}\right)\right\|_{\mathcal{B}_{l}^{m, 0}}=\left\|\left(\bar{\rho}_{t}+\bar{\rho} V+\bar{\rho}_{x} \tilde{u}\right) \omega-\bar{\rho} \tilde{u} \omega_{x}-\bar{\rho} \bar{v} \omega_{\eta}\right\|_{\mathcal{B}_{l}^{m, 0}}
$$

where

$$
\begin{gathered}
\left\|\left(\bar{\rho}_{t}+\bar{\rho} V\right) \omega\right\|_{\mathcal{B}_{l}^{m, 0}} \lesssim\|\omega\|_{\mathcal{B}_{l}^{m, 0}}, \\
\left\|\bar{\rho} \bar{v} \omega_{\eta}\right\|_{\mathcal{B}_{l}^{m, 0}} \lesssim\|\bar{v}\|_{L^{\infty}}\left\|\omega_{\eta}\right\|_{\mathcal{B}_{l}^{m, 0}}+\|\bar{v}\|_{\mathcal{D}_{c o, 0}^{m, 0}}\left\|\omega_{\eta}\right\|_{L_{\eta, l}^{2}\left(L_{x}^{\infty}\right)},
\end{gathered}
$$


and

$$
\begin{aligned}
\left\|\bar{\rho} \tilde{u} \omega_{x}\right\|_{\mathcal{B}_{l}^{m, 0}} & =\left\|\bar{\rho}(\tilde{u}-U) \omega_{x}+\bar{\rho} U \omega_{x}\right\|_{\mathcal{B}_{l}^{m, 0}} \\
& \lesssim\|\tilde{u}-U\|_{\mathcal{B}_{l}^{m, 0}}\left\|\omega_{x}\right\|_{L^{\infty}}+\left(\|\tilde{u}-U\|_{L^{\infty}}+1\right)\|\omega\|_{\mathcal{B}_{l}^{m+1,0}} .
\end{aligned}
$$

The term $\left\|\bar{\rho}_{x} \tilde{u} \omega\right\|_{\mathcal{B}_{l}^{m, 0}}$ can be estimated similarly. Moreover, we have

$$
\|2 \chi \omega\|_{\mathcal{B}_{l}^{m, 1}} \lesssim\|\chi\|_{L^{\infty}}\|\omega\|_{\mathcal{B}_{l}^{m, 1}}+\|\chi\|_{\mathcal{D}_{0}^{m, 1}}\|\omega\|_{L_{\eta, l}^{2}\left(L_{x}^{\infty}\right)}
$$

And

$$
\left\|\bar{\rho} \partial_{\eta}\left(\xi \int_{0}^{\eta} \omega(t, x, \tilde{\eta}) d \tilde{\eta}\right)\right\|_{\mathcal{B}_{l}^{m, 0}}=\left\|\bar{\rho}\left(\xi_{\eta} \int_{0}^{\eta} \omega(t, x, \tilde{\eta}) d \tilde{\eta}+\xi \omega\right)\right\|_{\mathcal{B}_{l}^{m, 0}}
$$

where

$$
\begin{aligned}
\| \bar{\rho}\left(\xi_{\eta} \int_{0}^{\eta} \omega(t, x, \tilde{\eta}) d \tilde{\eta} \|_{\mathcal{B}_{l}^{m, 0}} \lesssim\right. & \left\|\xi_{1 \eta}\right\|_{\mathcal{B}_{l}^{m, 0}}\|\omega\|_{L_{\eta, l}^{2}\left(L_{x}^{\infty}\right)}+\left\|\xi_{1 \eta}\right\|_{L_{\eta, l}^{2}\left(L_{x, t}^{\infty}\right)}\|\omega\|_{\mathcal{B}_{l}^{m, 0}} \\
& +\|\tilde{u}-U\|_{\mathcal{B}_{l}^{m, 1}}\|\omega\|_{L_{\eta, l}^{2}\left(L_{x}^{\infty}\right)}+\|\tilde{u}\|_{L_{l}^{\infty}}\|\omega\|_{\mathcal{B}_{l}^{m, 1}}
\end{aligned}
$$

and

$$
\|\xi \omega\|_{\mathcal{B}_{l}^{m, 0}} \lesssim\left\|\xi_{1}\right\|_{\mathcal{B}_{l}^{m, 0}}\|\omega\|_{L_{\eta, l}^{\infty}}+\left\|\xi_{1}\right\|_{L_{l}^{\infty}}\|\omega\|_{\mathcal{B}_{l}^{m, 0}}+\|\tilde{u}-U\|_{\mathcal{B}_{l}^{m, 0}}\|\omega\|_{L^{\infty}}+\left(\|\tilde{u}\|_{L^{\infty}}+1\right)\|\omega\|_{\mathcal{B}_{l}^{m, 1}}
$$

Plugging the above estimates into (3.14) yields

$$
\|\omega\|_{\mathcal{B}_{l}^{m, 2}} \lesssim \lambda_{3,1}\left(\|\omega\|_{\mathcal{B}_{l}^{m, 1}}+\|\omega\|_{\mathcal{B}_{l}^{m+1,0}}\right)+\lambda_{m, 1}\|\omega\|_{\mathcal{B}_{l}^{3,1}}+\|\tilde{f}\|_{\mathcal{B}_{l}^{m, 1}}
$$

Next, for any fixed $n \geq 3$, applying the differential operator $Z^{m} \partial_{\eta}^{n-2}$ on the equation (3.13) gives

$$
\begin{aligned}
& \|\omega\|_{\mathcal{B}_{l}^{m, n}} \\
= & \left\|\bar{\rho}\left(\omega_{t}+(\tilde{u} \omega)_{x}+(\eta V \omega)_{\eta}+(\bar{v} \omega)_{\eta}-\frac{2}{\bar{\rho}}\left(\omega \frac{\partial_{\eta}^{2} \tilde{u}}{\partial_{\eta} \tilde{u}}\right)_{\eta}+\left(\xi \int_{0}^{\eta} \omega(t, x, \tilde{\eta}) d \tilde{\eta}\right)_{\eta}-\frac{\bar{\rho}_{t}}{\bar{\rho}} \omega-\tilde{f}_{\eta}\right)\right\|_{\mathcal{B}_{l}^{m, n-2}},
\end{aligned}
$$

where

$$
\begin{gathered}
\| \bar{\rho}\left(\omega_{t}+(\eta V \omega)_{\eta}-\frac{\bar{\rho}_{t}}{\bar{\rho}} \omega\left\|_{\mathcal{B}_{l}^{m, n-2}} \lesssim\right\| \omega \|_{\mathcal{B}_{l}^{m+1, n-2}},\right. \\
\left\|\bar{\rho}(\tilde{u} \omega)_{x}+\bar{\rho}(\bar{v} \omega)_{\eta}\right\|_{\mathcal{B}_{l}^{m, n-2}} \lesssim\|\omega\|_{\mathcal{B}_{l}^{m, n-2}}+\|\tilde{u}-U\|_{\mathcal{B}_{l}^{m, n-2}}\left\|\omega_{x}\right\|_{L^{\infty}}+\left(\|\tilde{u}-U\|_{L^{\infty}}+1\right)\|\omega\|_{\mathcal{B}_{l}^{m+1, n-2}} \\
+\|\bar{v}\|_{L^{\infty}}\left\|\omega_{\eta}\right\|_{\mathcal{B}_{l}^{m, n-2}}+\|\bar{v}\|_{\mathcal{D}_{0}^{m, n-2}}\left\|\omega_{\eta}\right\|_{L_{\eta, l}^{2}\left(L_{x}^{\infty}\right)}, \\
\left\|2(\omega \chi)_{\eta}\right\|_{\mathcal{B}_{l}^{m, n-2}} \lesssim\|\omega\|_{\mathcal{B}_{l}^{m, n-1}}\|\chi\|_{L^{\infty}}+\|\chi\|_{\mathcal{D}_{0}^{m, n-1}}\|\omega\|_{L_{\eta, l}^{2}\left(L_{x}^{\infty}\right)},
\end{gathered}
$$


and

$$
\begin{aligned}
&\left\|\left(\xi \int_{0}^{\eta} \omega(t, x, \tilde{\eta}) d \tilde{\eta}\right)_{\eta}\right\|_{\mathcal{B}_{l}^{m, n-2}}=\left\|\xi_{\eta} \int_{0}^{\eta} \omega(t, x, \tilde{\eta}) d \tilde{\eta}+\xi \omega\right\|_{\mathcal{B}_{l}^{m, n-2}} \\
& \lesssim\left\|\xi_{\eta}\right\|_{\mathcal{B}_{l}^{m, n-2}}\|\omega\|_{L_{\eta, l}^{2}\left(L_{x}^{\infty}\right)}+\left\|\xi_{\eta}\right\|_{L_{l}^{\infty}}\|\omega\|_{\mathcal{B}_{l}^{m, n-2}}+\|\xi\|_{\mathcal{B}_{l}^{m, n-2}}\|\omega\|_{L_{\eta, l}^{\infty}}+\|\xi\|_{L_{l}^{\infty}}\|\omega\|_{\mathcal{B}_{l}^{m, n-2}} .
\end{aligned}
$$

Thus, we obtain

$$
\|\omega\|_{\mathcal{B}_{l}^{m, n}} \lesssim\left(\lambda_{3,1}+1\right)\left(\|\omega\|_{\mathcal{B}_{l}^{m+1, n-2}}+\|\omega\|_{\mathcal{B}_{l}^{m, n-1}}\right)+\lambda_{m, n-1}\|\omega\|_{\mathcal{B}_{l}^{3,1}}+\|\tilde{f}\|_{\mathcal{B}_{l}^{m, n-1}}
$$

By induction on $n$, we conclude the estimate (3.5). And this completes the proof of Theorem 3.1 .

\section{Iteration scheme and convergence}

Based on the energy estimate (3.5) on the solution to the linearized equations obtained in the previous section, we now study the well-posedness of the nonlinear problem (1.1) by using a suitable linear iteration scheme. From (3.5), there is a loss of regularity in the solutions to the linearized problem (3.1) with respect to both of the background states and initial data. Hence, as in [1, we apply the Nash-Moser-Hömander iteration scheme. As we explained in Section 1 , we do not have the divergence free condition, and the far-field state is not uniform. Thus, the shear flow is no longer the special exact solution to the compressible Prandtl equations (1.1) in contrast to the incompressible problem studied in [1]. Thus, to start the Nash-MoserHömander iteration, we need to construct a proper zero-th order approximate solution satisfying the nonlinear compressible Prandtl equations with enough decay in $\eta$. The construction will be given Subsection 4.1. Then, in Subsection 4.2 we present the Nash-Moser-Hömander iteration scheme for the problem (1.1). The estimates of the approximate solutions are obtained in Subsection 4.3. In Subsection 4.4, we conclude the convergence of iteration for the existence and uniqueness of the solution to the nonlinear problem (1.1)-(1.3).

\subsection{The Zero-th order approximate solution}

In this subsection, we construct the initial approximate solution to in the following three subsections.

\subsubsection{Compatibility conditions and initial data}

Set

$$
u=U(t, x)+\bar{u}, \quad v=V(t, x) \eta+\bar{v} .
$$

By using the Bernoulli law (1.4), from (1.1) we know that $(\bar{u}, \bar{v})$ satisfies

$$
\left\{\begin{array}{l}
\bar{u}_{t}+U_{x} \bar{u}+U \bar{u}_{x}+\bar{u} \bar{u}_{x}+(\bar{v}+V \eta) \bar{u}_{\eta}-\frac{1}{\bar{\rho}} \partial_{\eta}^{2} \bar{u}=0 \\
\partial_{\eta}(\bar{\rho} \bar{v})+\partial_{x}(\bar{\rho} \bar{u})=0 \\
\left.\bar{u}(t, x, \eta)\right|_{t=0}=u_{0}-U(0, x),\left.\quad \bar{v}\right|_{\eta=0}=0
\end{array}\right.
$$


Denote

$$
\bar{u}^{j}(x, \eta)=\left.\partial_{t}^{j} \bar{u}(t, x, \eta)\right|_{t=0}, \quad \bar{v}^{j}(x, \eta)=\left.\partial_{t}^{j} \bar{v}(t, x, \eta)\right|_{t=0} .
$$

From the compatibility condition of (4.1), $\left\{\bar{u}^{j}, \bar{v}^{j}\right\}_{j \leq k_{0}}$ is in turn given explicitly by $u_{0}(x, \eta), U(0, z)$ and $V(0, x)$.

We define the first approximate solution $(\bar{u}, \bar{v})$ of (4.1) as follows.

$$
u^{a}(t, x, \eta)=\sum_{j=0}^{k_{0}} \frac{t^{j}}{j !} \bar{u}^{j}(x, \eta), \quad v^{a}(t, x, \eta)=-\frac{1}{\bar{\rho}} \int_{0}^{\eta}\left(\bar{\rho} u^{a}\right)_{x}(t, x, \tilde{\eta}) d \tilde{\eta} .
$$

From (H3) and (H4) in the Main Assumptions (H), it follows that

$$
\max _{0 \leq t \leq T}\left\|\langle\eta\rangle^{\gamma+\alpha_{2}} D^{\alpha} u^{a}(t, \cdot)\right\|_{L^{2}\left(\mathbb{T} \times \mathbb{R}^{+}\right)} \leq C C_{0}, \quad|\alpha| \leq 2 k_{0}
$$

for a fixed $T>0$, where $C$ depends on $\sigma_{0}$ and the Sobolev norms of $\partial_{t}^{k}\left(\bar{\rho}, U, V, U_{x}\right), k \leq k_{0}$. Setting

$$
u^{a 1}(t, x, \eta)=U(t, x)+u^{a}(t, x, \eta), \quad v^{a 1}(t, x, \eta)=V(t, x) \eta+v^{a}(t, x, \eta),
$$

then, $\left(u^{a 1}, v^{a 1}\right)$ is an approximate solution to the problem (1.1) satisfying compatibility conditions up to order $k_{0}$ and initial data.

\subsubsection{Improving decay in $\eta$}

Note that the approximate solution $\left(u^{a 1}, v^{a 1}\right)$ satisfies

$$
\lim _{\eta \rightarrow+\infty} u^{a 1}(t, x, \eta)=U(t, x),
$$

and the divergence constraint

$$
\partial_{x}\left(\bar{\rho} u^{a 1}\right)+\partial_{\eta}\left(\bar{\rho} v^{a 1}\right)=-\bar{\rho}_{t},
$$

for all $t \geq 0$. However, the error

$$
f^{a 1}=\left(\partial_{t}+u^{a 1} \partial_{x}+v^{a 1} \partial_{\eta}-\frac{1}{\bar{\rho}(t, x)} \partial_{\eta}^{2}\right) u^{a 1}+P_{x}
$$

does not have enough decay compared with $\partial_{\eta} u^{a 1}$ as $\eta \rightarrow+\infty$. Since this property is essential for the convergence of the Nash-Moser-Hörmander iteration scheme of the nonlinear problem given in next section, we need to modify the approximate solution $\left(u^{a 1}, v^{a 1}\right)$ as follows.

From (1.1), $\partial_{\eta} u$ satisfies

$$
\left\{\begin{array}{l}
\left(\partial_{t}+u \partial_{x}+v \partial_{\eta}-\frac{1}{\bar{\rho}(t, x)} \partial_{\eta}^{2}\right) u_{\eta}+\left(u_{x}+v_{\eta}\right) u_{\eta}=0 \\
\left.u_{\eta}\right|_{\eta=0}=\bar{\rho} P_{x}
\end{array}\right.
$$


This motivates us to consider the following initial-boundary value problem for a linear degenerate parabolic equation:

$$
\left\{\begin{array}{l}
\phi_{t}+\left(u^{a 1} \phi\right)_{x}+\left(v^{a 1} \phi\right)_{\eta}-\frac{1}{\bar{\rho}} \partial_{\eta}^{2} \phi=0 \\
\left.\partial_{\eta} \phi\right|_{\eta=0}=\left(\bar{\rho} P_{x}\right)(t, x) \\
\left.\phi\right|_{t=0}=\left(\partial_{\eta} u_{0}\right)(x, \eta)
\end{array}\right.
$$

Suppose that the solution $\phi$ of (4.7) is obtained. Define an approximate solution $\left(u^{a 2}, v^{a 2}\right)$ as

$$
u^{a 2}=U(t, x)-\int_{\eta}^{\infty} \phi(t, x, \tilde{\eta}) d \tilde{\eta}, \quad v^{a 2}=V \eta+\frac{1}{\bar{\rho}} \int_{0}^{\eta}\left(\bar{\rho} \int_{\tilde{\eta}}^{\infty} \phi(t, x, s) d s\right)_{x} d \tilde{\eta} .
$$

It is straightforward to verify that the compatibility conditions of (1.1), the far-field condition (4.5) and the divergence constraint (4.6) still hold for $\left(u^{a 2}, v^{a 2}\right)$. Moreover, it satisfes the equation,

$$
u_{t}^{a 2}+u^{a 2} u_{x}^{a 2}+v^{a 2} u_{\eta}^{a 2}+P_{x}-\frac{1}{\bar{\rho}} \partial_{\eta}^{2} u^{a 2}=f^{0}
$$

where

$$
f^{0}=-\int_{\eta}^{\infty}\left(\left[U-u^{a 1}-\int_{\tilde{\eta}}^{\infty} \phi(t, x, s) d s\right] \phi\right)_{x} d \tilde{\eta}-\left(v^{a 2}-v^{a 1}\right) \phi
$$

which will be shown to decay faster than $\partial_{\eta} u^{a 2}$.

From the boundedness of $u^{a}$ given in (4.3) and some elementary weighted energy estimates on the solution to (4.7), we have

Proposition 4.1 Under the Main Assumptions (H) on the initial data, there exists a unique solution $\phi(t, x, \eta)$ to (4.7). Moreover, there is $T>0$ such that $\phi$ satisfies

$$
\left\{\begin{array}{l}
\max _{0 \leq t \leq T}\|\phi(t)\|_{H_{\gamma}^{2 k_{0}}} \leq C_{1}, \quad \phi(t, x, \eta) \geq \frac{C_{2}}{(1+\eta)^{\gamma+2}}, \quad \forall(t, x, \eta) \in[0, T] \times \mathbb{T} \times \mathbb{R}^{+}, \\
\left\|(1+\eta)^{\gamma+2+\alpha_{2}} D^{\alpha} \phi\right\|_{L^{\infty}\left([0, T] \times \mathbb{T} \times \mathbb{R}^{+}\right)} \leq C_{3}, \quad|\alpha| \leq k_{0},
\end{array}\right.
$$

where

$$
\|\phi(t)\|_{H_{\gamma}^{2 k_{0}}}=\sum_{|\alpha| \leq 2 k_{0}}\left\|(1+\eta)^{\gamma+\alpha_{2}} D^{\alpha} \phi(t)\right\|_{L^{2}\left(\mathbb{T} \times \mathbb{R}^{+}\right)},
$$

with $D^{\alpha}=\partial_{t, x}^{\alpha_{1}} \partial_{\eta}^{\alpha_{2}}, \alpha=\left(\alpha_{1}, \alpha_{2}\right)$.

Proof. The proof is divided in two steps.

(1) Applying the operator $D^{\alpha}=\partial_{t, x}^{\alpha_{1}} \partial_{\eta}^{\alpha_{2}}$ to the equation (4.7), multiplying the resulting equation by $(1+\eta)^{2 \gamma+2 \alpha_{2}} D^{\alpha} \phi$ and integrating it over $\mathbb{T} \times \mathbb{R}^{+}$, we obtain

$$
\frac{1}{2} \frac{d}{d t}\left\|(1+\eta)^{\gamma+\alpha_{2}} D^{\alpha} \phi(t)\right\|_{L^{2}}^{2}+\left\|\frac{1}{\sqrt{\bar{\rho}}}(1+\eta)^{\gamma+\alpha_{2}} D^{\alpha} \phi_{\eta}(t)\right\|_{L^{2}}^{2}=\sum_{i=1}^{7} I_{i}
$$


where

$$
\begin{gathered}
I_{1}=\int_{\mathbb{T} \times \mathbb{R}^{+}}\left(u_{x}^{a 1}+v_{\eta}^{a 1}\right)(1+\eta)^{2 \gamma+2 \alpha_{2}}\left(D^{\alpha} \phi\right)^{2} d x d \eta, \\
I_{2}=\int_{\mathbb{T} \times \mathbb{R}^{+}}\left(u^{a 1} D^{\alpha} \phi_{x}+v^{a 1} D^{\alpha} \phi_{\eta}\right)(1+\eta)^{2 \gamma+2 \alpha_{2}} D^{\alpha} \phi d x d \eta, \\
I_{3}=2\left(\gamma+\alpha_{2}\right) \int_{\mathbb{T} \times \mathbb{R}^{+}} \frac{1}{\bar{\rho}}(1+\eta)^{2 \gamma+2 \alpha_{2}-1}\left(D^{\alpha} \phi_{\eta}\right)\left(D^{\alpha} \phi\right) d x d \eta, \\
I_{4}=\sum_{0<\beta \leq \alpha} C_{\alpha}^{\beta} \int_{\mathbb{T} \times \mathbb{R}^{+}}(1+\eta)^{2 \gamma+2 \alpha_{2}} D^{\beta}\left(u_{x}^{a 1}+v_{\eta}^{a 1}\right)\left(D^{\alpha-\beta} \phi\right)\left(D^{\alpha} \phi\right) d x d \eta, \\
I_{5}=\sum_{0<\beta \leq \alpha} C_{\alpha}^{\beta} \int_{\mathbb{T} \times \mathbb{R}^{+}}(1+\eta)^{2 \gamma+2 \alpha_{2}}\left(D^{\beta} u^{a 1} D^{\alpha-\beta} \phi_{x}+D^{\beta} v^{a 1} D^{\alpha-\beta} \phi_{\eta}\right) D^{\alpha} \phi d x d \eta, \\
I_{6}=\sum_{0<\beta \leq \alpha, \beta_{2}=0} C_{\alpha}^{\beta} \int_{\mathbb{T} \times \mathbb{R}^{+}}(1+\eta)^{2 \gamma+2 \alpha_{2}}\left(D^{\beta} \frac{1}{\bar{\rho}}\right)\left(\partial_{\eta}^{2} D^{\alpha-\beta} \phi\right)\left(D^{\alpha} \phi\right) d x d \eta,
\end{gathered}
$$

and

$$
I_{7}=\left.\int_{\mathbb{T}} \frac{1}{\bar{\rho}}\left(\partial_{\eta} D^{\alpha} \phi D^{\alpha} \phi\right)\right|_{\eta=0} d x .
$$

It is straightforward to show

$$
\begin{gathered}
\left|I_{1}\right| \leq\left\|u_{x}^{a 1}, v_{\eta}^{a 1}\right\|_{L^{\infty}}\left\|(1+\eta)^{\gamma+\alpha_{2}} D^{\alpha} \phi\right\|_{L^{2}}^{2}, \\
\left|I_{2}\right|=\left|\int_{\mathbb{T}^{a \mathbb{R}^{+}}}\left(u^{a 1} D^{\alpha} \phi_{x}+v^{a 1} D^{\alpha} \phi_{\eta}\right)(1+\eta)^{2 \gamma+2 \alpha_{2}} D^{\alpha} \phi d x d \eta\right| \\
\lesssim\left(\left\|u_{x}^{a 1}\right\|_{L^{\infty}}+\left\|v_{\eta}^{a 1}\right\|_{L^{\infty}}+\left\|v_{\eta}^{a 1} /(1+\eta)\right\|_{L^{\infty}}\right)\left\|(1+\eta)^{\gamma+\alpha_{2}} D^{\alpha} \phi\right\|_{L^{2}}^{2},
\end{gathered}
$$

by integration by parts, and using $\left.v^{a 1}\right|_{\eta=0}=0$, and

$$
\begin{aligned}
\left|I_{3}\right| & =2\left(\gamma+\alpha_{2}\right)\left|\int_{\mathbb{T} \times \mathbb{R}^{+}} \frac{1}{\bar{\rho}} D^{\alpha} \phi_{\eta}(1+\eta)^{2 \gamma+2 \alpha_{2}-1} D^{\alpha} \phi d x d \eta\right| \\
& \leq \frac{1}{8}\left\|\frac{1}{\sqrt{\bar{\rho}}}(1+\eta)^{\gamma+\alpha_{2}} D^{\alpha} \phi_{\eta}\right\|_{L^{2}}^{2}+C\left\|\frac{1}{\bar{\rho}}\right\|_{L^{\infty}}\left\|(1+\eta)^{\gamma+\alpha_{2}} D^{\alpha} \phi\right\|_{L^{2}}^{2} .
\end{aligned}
$$

On the other hand, we have

$$
\begin{aligned}
\left|I_{4}\right| & =\left|\sum_{0<\beta \leq \alpha} C_{\alpha}^{\beta} \int_{{\mathbb{T} \times \mathbb{R}^{+}}}(1+\eta)^{\beta_{2}} D^{\beta}\left(\frac{-\bar{\rho}_{t}-\bar{\rho}_{x} u^{a 1}}{\bar{\rho}}\right)(1+\eta)^{\gamma+\alpha_{2}-\beta_{2}} D^{\alpha-\beta} \phi(1+\eta)^{\gamma+\alpha_{2}} D^{\alpha} \phi d x d \eta\right| \\
& \leq C\left\|\sum_{\beta} D^{\beta}\left(\frac{\bar{\rho}_{t}+\bar{\rho}_{x} U}{\bar{\rho}}\right)\right\|_{L^{\infty}}\|\phi\|_{H_{\gamma}^{2 k_{0}}}^{2}+\left|\tilde{I}_{4}\right|,
\end{aligned}
$$

by noting that $\beta_{2}=0$ for the operator $D^{\beta}$ acting on $\frac{\bar{\rho}_{t}+\bar{\rho}_{x} U}{\bar{\rho}}$. Hence,

$$
\left|\tilde{I}_{4}\right| \leq C\left\|(1+\eta)^{\beta_{2}}\left(D^{\beta} \frac{\bar{\rho}_{x}\left(u^{a 1}-U\right)}{\bar{\rho}}\right)\right\|_{L^{\infty}}\|\phi\|_{H_{\gamma}^{2 k_{0}}}^{2} \text {, for }|\beta| \leq k_{0},
$$


and

$$
\begin{aligned}
\left|\tilde{I}_{4}\right| & \leq C\left\|(1+\eta)^{\beta_{2}}\left(D^{\beta} \frac{\bar{\rho}_{x}\left(u^{a 1}-U\right)}{\bar{\rho}}\right)\right\|_{L^{2}}\left\|(1+\eta)^{\gamma+\alpha_{2}-\beta_{2}} D^{\alpha-\beta} \phi\right\|_{L^{\infty}}\left\|(1+\eta)^{\gamma+\alpha_{2}} D^{\alpha} \phi\right\|_{L^{2}} \\
& \leq C\left\|(1+\eta)^{\beta_{2}}\left(D^{\beta} \frac{\bar{\rho}_{x}\left(u^{a 1}-U\right)}{\bar{\rho}}\right)\right\|_{L^{2}}\|\phi\|_{H_{\gamma}^{2 k_{0}}}^{2}, \text { for }|\beta|>k_{0},
\end{aligned}
$$

by using the weighted Sobolev embedding. Similarly, one has

$$
\begin{aligned}
\left|I_{5}\right| & =\left|\sum_{0<\beta \leq \alpha} \int_{\mathbb{T} \times \mathbb{R}^{+}} C_{\alpha}^{\beta}\left(D^{\beta} u^{a 1} D^{\alpha-\beta} \phi_{x}+D^{\beta} v^{a 1} D^{\alpha-\beta} \phi_{\eta}\right)(1+\eta)^{2 \gamma+2 \alpha_{2}} D^{\alpha} \phi d x d \eta\right| \\
& \leq\left|I_{5}^{1}\right|+\left|I_{5}^{2}\right|,
\end{aligned}
$$

where

$$
\left|I_{5}^{1}\right| \leq\left\{\begin{array}{l}
C\left(\left\|D^{\beta}\left(u^{a 1}-U\right)(1+\eta)^{\beta_{2}}\right\|_{L^{\infty}}+\left\|D^{\beta} U\right\|_{L^{\infty}}\right)\|\phi\|_{H_{\gamma}^{2 k_{0}}}^{2}, \quad \text { for } 1 \leq|\beta| \leq k_{0}, \\
C\left(\left\|D^{\beta}\left(u^{a 1}-U\right)(1+\eta)^{\beta_{2}}\right\|_{L^{2}}+\left\|D^{\beta} U\right\|_{L^{\infty}}\right)\|\phi\|_{H_{\gamma}^{2 k_{0}}}^{2}, \text { for }|\beta|>k_{0},
\end{array}\right.
$$

and

$$
\begin{gathered}
\left|I_{5}^{2}\right| \leq\left\{\begin{array}{l}
C\left\|D^{\beta} v^{a 1}(1+\eta)^{\beta_{2}-1}\right\|_{L^{\infty}}\|\phi\|_{H_{\gamma}^{2 k_{0}}}^{2}, \text { for } 1 \leq|\beta| \leq k_{0}, \\
C\left\|D^{\beta} v^{a 1}(1+\eta)^{\beta_{2}-1}\right\|_{L^{2}}\|\phi\|_{H_{\gamma}^{2 k_{0}}}^{2}, \text { for } \beta_{2} \neq 0,|\beta|>k_{0} ;
\end{array}\right. \\
\left|I_{5}^{2}\right| \leq C\left\|D^{\beta} v^{a 1}(1+\eta)^{-1}\right\|_{L_{\eta}^{\infty}\left(L_{x}^{2}\right)}\left\|D^{\alpha-\beta} \phi_{\eta}(1+\eta)^{\gamma+\alpha_{2}+1}\right\|_{L_{\eta}^{2}\left(L_{x}^{\infty}\right)}\left\|D^{\alpha} \phi(1+\eta)^{\gamma+\alpha_{2}}\right\|_{L^{2}}, \\
\leq C\left\|D^{\beta} v^{a 1}(1+\eta)^{-1}\right\|_{L_{\eta}^{\infty}\left(L_{x}^{2}\right)}\|\phi\|_{H_{\gamma}^{2 k_{0}}}^{2} \text { for } \beta_{2}=0,|\beta|>k_{0} .
\end{gathered}
$$

For the term $I_{6}$, by integration by parts, we have

$$
\begin{aligned}
\left|I_{6}\right|= & \sum_{0<\beta \leq \alpha, \beta_{2}=0}\left(\mid \int_{\mathbb{T} \times \mathbb{R}^{+}} C_{\alpha}^{\beta}\left(2 \gamma+2 \alpha_{2}\right)(1+\eta)^{2 \gamma+2 \alpha_{2}-1}\left(D^{\beta} \frac{1}{\bar{\rho}}\right)\left(\partial_{\eta} D^{\alpha-\beta} \phi\right)\left(D^{\alpha} \phi\right) d x d \eta\right. \\
& +\int_{\mathbb{T} \times \mathbb{R}^{+}} C_{\alpha}^{\beta}(1+\eta)^{2 \gamma+2 \alpha_{2}}\left(D^{\beta} \frac{1}{\bar{\rho}}\right)\left(\partial_{\eta} D^{\alpha-\beta} \phi\right)\left(D^{\alpha} \phi_{\eta}\right) d x d \eta \mid \\
& \left.-\left|\int_{\mathbb{T}} C_{\alpha}^{\beta}\left(D^{\beta} \frac{1}{\bar{\rho}}\right)\left(\partial_{\eta} D^{\alpha-\beta} \phi\right)\left(D^{\alpha} \phi\right)\right|_{\eta=0} d x \mid\right) \\
\leq & \sum_{0<\beta \leq \alpha, \beta_{2}=0}\left(\left|\int_{\mathbb{T}} C_{\alpha}^{\beta}\left(D^{\beta} \frac{1}{\bar{\rho}}\right)\left(\partial_{\eta} D^{\alpha-\beta} \phi\right)\left(D^{\alpha} \phi\right)\right|_{\eta=0} d x \mid+C\left\|(1+\eta)^{\gamma+\alpha_{2}} D^{\alpha-\beta} \phi_{\eta}\right\|_{L^{2}}^{2}\right) \\
& +\frac{1}{8}\left\|\frac{1}{\sqrt{\bar{\rho}}}(1+\eta)^{\gamma+\alpha_{2}} D^{\alpha} \phi_{\eta}\right\|_{L^{2}}^{2} .
\end{aligned}
$$

It remains to handle the boundary integration terms on the right hand side of the above estimate and $I_{7}$. For illustration, we only estimate $I_{7}$.

Firstly, noticing that

$$
\left.\partial_{\eta} \phi\right|_{\eta=0}=\bar{\rho} P_{x}
$$


and applying the operator $\partial_{\eta}$ on the equation (4.7), we obtain

$$
\frac{1}{\bar{\rho}} \partial_{\eta}^{3} \phi=\left(\phi_{\eta}\right)_{t}+\left(u_{x}^{a 1} \phi\right)_{\eta}+\left(u^{a 1} \phi_{x}\right)_{\eta}+\left(v^{a 1} \phi\right)_{\eta \eta} .
$$

Taking this equation on the boundary $\{\eta=0\}$ and using the boundary condition, we get

$$
\left.\frac{1}{\bar{\rho}} \partial_{\eta}^{3} \phi\right|_{\eta=0}=\left(\bar{\rho} P_{x}\right)_{t}+u_{x}^{a 1} \bar{\rho} P_{x}+\left.u_{x \eta}^{a 1} \phi\right|_{\eta=0}+u^{a 1}\left(\bar{\rho} P_{x}\right)_{x}+\left.u_{\eta}^{a 1} \phi_{x}\right|_{\eta=0}+2 v_{\eta}^{a 1} \bar{\rho} P_{x}+\left.v_{\eta \eta}^{a 1} \phi\right|_{\eta=0} .
$$

By induction, for positive integer $k$, we have

$$
\begin{aligned}
\left.\frac{1}{\bar{\rho}} \partial_{\eta}^{2 k+1} \phi\right|_{\eta=0}= & \left(\partial_{\eta}^{2 k-1} \phi\right)_{t}+\partial_{\eta}^{2 k-1}\left(u_{x}^{a 1} \phi\right)+\partial_{\eta}^{2 k-1}\left(u^{a 1} \phi_{x}\right)+\partial_{\eta}^{2 k}\left(v^{a 1} \phi\right) \\
= & {\left[F\left(D_{|\alpha| \leq 2 k-2}^{\alpha} u^{a 1}, D_{|\beta| \leq 2 k-2}^{\beta} v^{a 1}, D_{|\gamma| \leq 2 k-3}^{\gamma} \phi, D^{\pi}\left(\bar{\rho}, P_{x}\right)\right)\right]_{t}+\sum_{i=1}^{2 k-1} C_{2 k-1}^{i} \partial_{\eta}^{i} u_{x}^{a 1} \partial_{\eta}^{2 k-1-i} \phi } \\
& +\sum_{j=1}^{2 k-1} C_{2 k-1}^{j} \partial_{\eta}^{i} u^{a 1} \partial_{\eta}^{2 k-1-j} \phi_{x}+\sum_{s=1}^{2 k} C_{2 k}^{s} \partial_{\eta}^{s} v^{a 1} \partial_{\eta}^{2 k-s} \phi \\
= & \left.G\left(D_{|\alpha| \leq 2 k}^{\alpha} u^{a 1}, D_{|\beta| \leq 2 k}^{\beta} v^{a 1}, D_{|\gamma| \leq 2 k-1}^{\gamma} \phi, D^{\pi}\left(\bar{\rho}, P_{x}\right)\right)\right),
\end{aligned}
$$

where $F, G$ are polynomial functions. Hence, the normal derivative of $\phi$ can be reduced by two order using the boundary condition and the equation (4.7). Therefore, we can use the trace estimate to control the boundary integral.

Thus, by summarizing the above estimates, and taking summation over $|\alpha| \leq 2 k_{0}$ for (4.11), it follows

$$
\frac{d}{d t}\|\phi(t)\|_{H_{\gamma}^{2 k_{0}}}^{2}+\sum_{|\alpha| \leq 2 k_{0}}\left\|\frac{1}{\sqrt{\bar{\rho}}}(1+\eta)^{\gamma+\alpha_{2}} D^{\alpha} \phi_{\eta}(t)\right\|_{L^{2}}^{2} \leq C\|\phi(t)\|_{H_{\gamma}^{2 k_{0}}}^{2} .
$$

which implies the first boundedness estimate given in (4.10) by using Gronwall inequality.

(2) Next, we apply the maximal principle to prove the second estimate given in (4.10).

From (4.7), $y(t, x, \eta) \triangleq(1+\eta)^{\gamma+2} \phi$ satisfies the following degenerate parabolic equation,

$$
y_{t}+\left(u_{x}^{a 1}+v_{\eta}^{a 1}-\frac{v^{a 1}(2+\gamma)}{1+\eta}-\frac{(\gamma+2)(\gamma+3)}{\bar{\rho}(1+\eta)^{2}}\right) y+u^{a 1} y_{x}+\left(v^{a 1}+\frac{2(\gamma+2)}{\bar{\rho}(1+\eta)}\right) y_{\eta}-\frac{1}{\bar{\rho}} \partial_{\eta}^{2} y=0 .
$$

By the maximal principle (see also Lemma E.2 in [11]), we have

$$
\min _{\mathbb{T} \times \mathbb{R}^{+}} y(t) \geq\left(1-\lambda t e^{\lambda t}\right) k(t),
$$

with

$$
k(t)=\min \left\{\left.\min _{\mathbb{T} \times \mathbb{R}^{+}} y\right|_{t=0},\left.\min _{[0, t] \times \mathbb{T}} y\right|_{\eta=0}\right\},
$$

for a fixed $\lambda \geq\left\|\left(u_{x}^{a 1}+v_{\eta}^{a 1}-\frac{v^{a 1}(2+\gamma)}{1+\eta}-\frac{(\gamma+2)(\gamma+3)}{\bar{\rho}(1+\eta)^{2}}\right)\right\|_{L^{\infty}}$. 
It follows from the Main Assumptions (H2) on the initial data that

$$
\min _{\mathbb{T} \times \mathbb{R}^{+}} y(0) \geq \sigma_{0}>0 .
$$

It suffices to derive the lower bound on $\left.\min _{[0, t] \times \mathbb{T}} y\right|_{\eta=0}$. Notice that $\left.y\right|_{\eta=0}=\left.\phi\right|_{\eta=0}$, the first boundedness estimate of (4.10) and the Sobolev inequality give

$$
\left\|\left.\phi_{t}\right|_{\eta=0}\right\|_{L^{\infty}} \leq C
$$

Consequently,

$$
\left.\phi(t)\right|_{\eta=0} \geq\left.\phi(0, x, \eta)\right|_{\eta=0}-C t \geq \sigma_{0}-C t .
$$

Thus, we have the lower bound given in the second estimate in (4.10) provided that $t$ is suitably small. The third estimate in (4.10) can also be proved by the maximal principle similarly (also refer to Lemma E.1 in [1] ). Then the proof of this proposition is completed.

\subsubsection{Boundary condition}

It is noted that the approximate solution $u^{a 2}$ does not satisfy the original boundary condition, that is, $\left.u^{a 2}\right|_{\eta=0} \neq 0$. For this, set

$$
\left.\zeta(t, x) \triangleq u^{a 2}\right|_{\eta=0}=U(t, x)-\int_{0}^{\infty} \phi(t, x, \eta) d \eta .
$$

$\zeta(t, x)$ is uniformly continuous and bounded due to (4.10). By the compatibility condition of the initial data, we have $\zeta(0, x)=0$. Consequently, $|\zeta(t, x)| \leq \varepsilon_{0}, t \in\left[0, t_{0}\right]$, with $\varepsilon_{0} \rightarrow 0$ as $t_{0}$ tends to zero.

In addition, there exists a smooth monotone decreasing function $\psi(\eta) \subseteq[0,1], \eta \geq 0$ such that $\operatorname{supp} \psi \subseteq[0,1], \psi(0)=1$ and $\left|\psi^{\prime}(\eta)\right|<C$. Note that there exists a positive constant $a_{0}$ such that $\phi(t, x, \eta)>a_{0}, \eta \in[0,1]$.

Now, define

$$
u^{a 3}=u^{a 2}-\zeta(t, x) \psi(\eta), \quad v^{a 3}=-\frac{1}{\bar{\rho}} \int_{0}^{\eta}\left(\bar{\rho}\left(u^{a 2}(t, x, \tilde{\eta})-U(x, t)-\zeta(t, x) \psi(\tilde{\eta})\right)_{x} d \tilde{\eta} .\right.
$$

It is direct to check that $\left.u^{a 3}(t, x, \eta)\right|_{\eta=0}=0$, and

$$
u_{\eta}^{a 3}(t, x, \eta)=\phi(t, x, \eta)-\zeta(t, x) \psi^{\prime}(\eta)>\frac{\phi}{2}>0
$$

provided that $t \in\left[0, t_{0}\right]$ with $t_{0}$ being suitably small. And the profile $\left(u^{a 3}, v^{a 3}\right)$ satisfies

$$
\left\{\begin{array}{l}
u_{t}^{a 3}+u^{a 3} u_{x}^{a 3}+v^{a 3} u_{\eta}^{a 3}+P_{x}-\frac{1}{\bar{\rho}} \partial_{\eta}^{2} u^{a 3}=f^{a} \\
\partial_{\eta}\left(\bar{\rho} v^{a 3}\right)+\partial_{x}\left(\bar{\rho} u^{a 3}\right)=-\bar{\rho}_{t}, \\
u^{a 3}(0, x, \eta)=\left(\partial_{\eta} u_{0}\right)(x, \eta)
\end{array}\right.
$$


with $f^{a}=f^{0}-\bar{f}^{0}$, where

$\bar{f}^{0}=\zeta_{t} \psi+\zeta \psi u_{x}^{a 2}+u^{a 2} \zeta_{x} \psi-\zeta \zeta_{x} \psi^{2}+v^{a 2} \zeta \psi^{\prime}-\frac{u_{\eta}^{a 2}(\bar{\rho} \zeta)_{x}}{\bar{\rho}} \int_{0}^{\eta} \psi(\tilde{\eta}) d \tilde{\eta}+\frac{\zeta \psi^{\prime}(\bar{\rho} \zeta)_{x}}{\bar{\rho}} \int_{0}^{\eta} \psi(\tilde{\eta}) d \tilde{\eta}-\frac{\psi^{\prime \prime} \zeta}{\bar{\rho}}$.

Remark 4.1 The approach of constructing the zero-th approximate solution to (1.1) introduced above can be applied to the incompressible Prandtl equations.

\subsection{The Nash-Moser-Hömander iteration scheme}

We now construct the approximate solution sequence of (1.1) by using the Nash-Moser-Hömander Iteration Scheme. The procedure mainly follows the one given in [1]. Thus, we will only present the main steps.

Denote the linearized operator $\mathcal{P}^{\prime}$ around $(\hat{\omega}, \hat{q})$ of (1.1) by

$$
\mathcal{P}_{(\hat{\omega}, \hat{q})}^{\prime}(\omega, q)=\partial_{t} \omega+\hat{\omega} \omega_{x}+\hat{q} \omega_{\eta}+\omega \hat{\omega}_{x}+q \hat{\omega}_{\eta}-\frac{1}{\bar{\rho}} \partial_{\eta}^{2} \omega
$$

Suppose that the approximate solutions $\left(u^{k}, v^{k}\right)$ of (1.1) have been constructed for all $k \leq n$, with $u^{0}=u^{a 3}$ and $v^{0}=v^{a 3}$ being defined in Subsection 4.1.3, we construct the $(n+1)$-th approximate solution $\left(u^{n+1}, v^{n+1}\right)$ as follows:

$$
u^{n+1}=u^{n}+\delta u^{n}=u^{a 3}+\tilde{u}^{n}+\delta u^{n}, \quad v^{n+1}=v^{n}+\delta v^{n}=v^{a 3}+\tilde{v}^{n}+\delta v^{n},
$$

where the increment $\left(\delta u^{n}, \delta v^{n}\right)$ is the solution to the following initial-boundary value problem

$$
\left\{\begin{array}{l}
\mathcal{P}_{\left(u_{\theta_{n}}^{n}, v_{\theta_{n}}^{n}\right)}^{\prime}\left(\delta u^{n}, \delta v^{n}\right)=f^{n}, \\
\partial_{\eta}\left(\bar{\rho}(t, x) \delta v^{n}\right)+\partial_{x}\left(\bar{\rho}(t, x) \delta u^{n}\right)=0, \\
\left.\delta u^{n}\right|_{\eta=0}=\left.\delta v^{n}\right|_{\eta=0}=0, \quad \lim _{\eta \rightarrow+\infty} \delta u^{n}=0, \\
\left.\delta u^{n}\right|_{t=0}=0 .
\end{array}\right.
$$

Here, $u_{\theta_{n}}^{n}=u^{a 3}+S_{\theta_{n}} \tilde{u}^{n}$ and $v_{\theta_{n}}^{n}=v^{a 3}+S_{\theta_{n}} \tilde{v}^{n}$ with $\theta_{n}=\sqrt{\theta_{0}^{2}+n}$ for any $n \geq 1$ and a large fixed constant $\theta_{0}$. The smoothing operator $S_{\theta}$ is defined by

$$
\left(S_{\theta} f\right)(t, x, \eta)=\iiint j_{\theta}(\tau) j_{\theta}(\xi) j_{\theta}(\mu) \tilde{f}\left(t-\tau+\theta^{-1}, x-\xi, \eta-\mu+\theta^{-1}\right) d \tau d \xi d \mu
$$

for a function $f$ defined on $\Omega=\left[0,+\infty\left[\times \mathbb{T}_{x} \times \mathbb{R}_{\eta}^{+}\right.\right.$with $\tilde{f}$ being the zero extension of $f$ to $\mathbb{R}^{3}$, and the mollifier $j_{\theta}(\tau)=\theta j(\theta \tau)$ with $j \in C_{0}^{\infty}(\mathbb{R})$ being a non-negative function satisfying Supp $j \subseteq[-1,1]$ and $\|j\|_{L^{1}}=1$.

In order to show that the approximate solution $\left(u^{n}, v^{n}\right)$ converges to the solution of the nonlinear problem (1.1), we need to define the source term $f^{n}$ properly for the problem (4.16).

To do this, denoting the nonlinear operator on the left hand side of (1.1) by $\mathcal{P}(\omega, q)$, obviously, the following identity holds:

$$
\mathcal{P}\left(u^{n+1}, v^{n+1}\right)-\mathcal{P}\left(u^{n}, v^{n}\right)=\mathcal{P}_{\left(u_{\theta_{n}}^{n}, v_{\theta_{n}}^{n}\right)}^{\prime}\left(\delta u^{n}, \delta v^{n}\right)+e_{n}
$$


where

$$
e_{n}=e_{n}^{1}+e_{n}^{2}
$$

with $e_{n}^{1}$ being the error term from the Newton iteration,

$$
\begin{aligned}
e_{n}^{1} & =\mathcal{P}\left(u^{n}+\delta u^{n}, v^{n}+\delta v^{n}\right)-\mathcal{P}\left(u^{n}, v^{n}\right)-\mathcal{P}_{\left(u^{n}, v^{n}\right)}^{\prime}\left(\delta u^{n}, \delta v^{n}\right) \\
& =\delta u^{n} \partial_{x}\left(\delta u^{n}\right)+\delta v^{n} \partial_{\eta}\left(\delta u^{n}\right),
\end{aligned}
$$

and $e_{n}^{2}$ being the error from mollifying the coefficients,

$$
\begin{aligned}
e_{n}^{2}= & \mathcal{P}_{\left(u^{n}, v^{n}\right)}^{\prime}\left(\delta u^{n}, \delta v^{n}\right)-\mathcal{P}_{\left(u_{\theta_{n}}^{n}, v_{\theta_{n}}^{n}\right)}^{\prime}\left(\delta u^{n}, \delta v^{n}\right) \\
= & \left(\left(1-S_{\theta_{n}}\right)\left(u^{n}-u^{a 3}\right)\right) \partial_{x}\left(\delta u^{n}\right)+\delta u^{n} \partial_{x}\left(\left(1-S_{\theta_{n}}\right)\left(u^{n}-u^{a 3}\right)\right) \\
& +\left(\left(1-S_{\theta_{n}}\right)\left(v^{n}-v^{a 3}\right)\right) \partial_{\eta} \delta u^{n}+\delta v^{n} \partial_{\eta}\left(\left(1-S_{\theta_{n}}\right)\left(u^{n}-u^{a 3}\right)\right) .
\end{aligned}
$$

Taking summation of (4.17) over all $n \in \mathbb{N}$ leads to

$$
\mathcal{P}\left(u^{n+1}, v^{n+1}\right)=\sum_{j=0}^{n}\left(\mathcal{P}_{\left(u_{\theta_{j}}^{j}, v_{\theta_{j}}^{j}\right)}^{\prime}\left(\delta u^{j}, \delta v^{j}\right)+e_{j}\right)+f^{a},
$$

with $f^{a}=\mathcal{P}\left(u^{a 3}, v^{a 3}\right)$.

It is obvious that if the approximate solution $\left(u^{n}, v^{n}\right)$ converges to the solution to (1.1), then the right hand side of (4.20) must converge to zero as $n$ tends to $+\infty$. In this way, it is convenient to require that $\left(\delta u^{n}, \delta v^{n}\right)(n \geq 0)$ satisfies the equation,

$$
\mathcal{P}_{\left(u_{\theta_{n}}^{n}, v_{\theta_{n}}^{n}\right)}^{\prime}\left(\delta u^{n}, \delta v^{n}\right)=f^{n},
$$

where $f^{n}$ is defined by

$$
\sum_{j=0}^{n} f^{j}=-S_{\theta_{n}}\left(\sum_{j=0}^{n-1} e_{j}\right)-S_{\theta_{n}} f^{a}
$$

inductively, that is,

$$
\left\{\begin{array}{l}
f^{0}=-S_{\theta_{0}} f^{a}, \quad f^{1}=\left(S_{\theta_{0}}-S_{\theta_{1}}\right) f^{a}+S_{\theta_{0}} f^{a}, \\
f^{n}=\left(S_{\theta_{n-1}}-S_{\theta_{n}}\right)\left(\sum_{j=0}^{n-2} e_{j}\right)-S_{\theta_{n}} e_{n-1}+\left(S_{\theta_{n-1}}-S_{\theta_{n}}\right) f^{a}, \quad \forall n \geq 2,
\end{array}\right.
$$

with $f^{a}$ given in (4.14).

We now give some properties of the smoothing operator in the following lemma, which also can be found in Section 4.1 of [1].

Lemma 4.1 The smoothing operator $\left\{S_{\theta}\right\}_{\theta>0}: \mathcal{A}_{l}^{0}(\Omega) \rightarrow \cap_{s \geq 0} \mathcal{A}_{l}^{s}(\Omega)$, satisfies the following estimates:

$$
\left\{\begin{array}{l}
\left\|S_{\theta} v\right\|_{\mathcal{A}_{l}^{s}} \leq C_{j} \theta^{(s-\alpha)_{+}}\|v\|_{\mathcal{A}_{l}^{\alpha}}, \quad \text { for all } s, \alpha \geq 0 \\
\left\|\left(1-S_{\theta}\right) v\right\|_{\mathcal{A}_{l}^{s}} \leq C_{j} \theta^{(s-\alpha)}\|v\|_{\mathcal{A}_{l}^{\alpha}}^{\alpha}, \quad \text { for all } 0 \leq s \leq \alpha
\end{array}\right.
$$

and

$$
\left\|\left(S_{\theta_{n}}-S_{\theta_{n-1}}\right) v\right\|_{\mathcal{A}_{l}^{s}} \leq C_{j} \triangle \theta_{n}^{s-\alpha}\|v\|_{\mathcal{A}_{l}^{\alpha}} \text {, for all } s, \alpha \geq 0,
$$

where $\triangle \theta_{n}=\theta_{n+1}-\theta_{n}$, and the constant $C_{j}$ depends only on the mollifier function $j \in C_{0}^{\infty}(\mathbb{R})$. 


\subsection{Estimates of the approximate solutions}

To study the solutions $\left(\delta u^{n}, \delta v^{n}\right)$ to the problem (4.16) with $f^{n}$ given in (4.22), as in Section 3, set

$$
\omega^{n}=\partial_{\eta}\left(\frac{\bar{\rho} \delta u^{n}}{\partial_{\eta} u_{\theta_{n}}^{n}}\right) .
$$

Then $\omega^{n}$ satisfies

$$
\left\{\begin{array}{l}
\partial_{t} \omega^{n}+\partial_{x}\left(u_{\theta_{n}}^{n} \omega^{n}\right)+\partial_{\eta}\left(v_{\theta_{n}}^{n} \omega^{n}\right)-\frac{2}{\bar{\rho}}\left(\omega^{n} \chi^{n}\right)_{\eta}+\left(\xi^{n} \int_{0}^{\eta} \omega^{n}(t, x, \tilde{\eta}) d \tilde{\eta}\right)_{\eta}-\frac{\bar{\rho}_{t}}{\bar{\rho}} \omega^{n}-\frac{1}{\bar{\rho}} \omega_{\eta \eta}^{n}=\tilde{f}_{\eta}^{n}, \\
\left.\frac{1}{\bar{\rho}}\left(\omega_{\eta}^{n}+2 \omega^{n} \chi^{n}\right)\right|_{\eta=0}=-\left.\tilde{f}^{n}\right|_{\eta=0}, \\
\left.\omega^{n}\right|_{t=0}=0
\end{array}\right.
$$

where

$$
\chi^{n}=\frac{\partial_{\eta}^{2} u_{\theta_{n}}^{n}}{\partial_{\eta} u_{\theta_{n}}^{n}}, \quad \xi^{n}=\frac{\left(\partial_{t}+u_{\theta_{n}}^{n} \partial_{x}+v_{\theta_{n}}^{n} \partial_{\eta}-\frac{1}{\bar{\rho}} \partial_{\eta}^{2}\right) \partial_{\eta} u_{\theta_{n}}^{n}}{\partial_{\eta} u_{\theta_{n}}^{n}}-\frac{u_{\theta_{n}}^{n} \bar{\rho}_{x}}{\bar{\rho}} \triangleq \xi_{1}^{n}-\xi_{2}^{n},
$$

and

$$
\tilde{f}^{n}=\frac{\bar{\rho} f^{n}}{\partial_{\eta} u_{\theta_{n}}^{n}}
$$

Similar to (3.3)-(3.4), we define

$$
\lambda_{k_{1}, k_{2}}=\left\|u_{\theta_{n}}^{n}-u^{a 3}\right\|_{\mathcal{B}_{l}^{k_{1}, k_{2}}}+\left\|Z^{k_{1}} \partial_{\eta}^{k_{2}} v_{\theta_{n}}^{n}\right\|_{L_{\eta}^{\infty}\left(L_{t, x}^{2}\right)}+\left\|Z^{k_{1}} \partial_{\eta}^{k_{2}} \chi^{n}\right\|_{L_{\eta}^{\infty}\left(L_{t, x}^{2}\right)}+\left\|\xi_{1}^{n}\right\|_{\mathcal{B}_{l}^{k_{1}, k_{2}}},
$$

and

$$
\lambda_{k}^{n}=\sum_{k_{1}+\left[\left(k_{2}+1\right) / 2\right] \leq k} \lambda_{k_{1}, k_{2}}^{n}
$$

Applying Theorem 3.1 to the linearized problem (4.26), we have

Theorem 4.1 Suppose the known functions $(\bar{\rho}, U, V)(t, x)$ satisfy the same assumptions as in Theorem [1.1, and the main assumptions (H) are satisfied. Then for any fixed $l>1 / 2$, the following estimate holds for the solution of the problem (4.26),

$$
\left\|\omega^{n}\right\|_{\mathcal{A}_{l}^{k}} \leq C_{1}\left(\lambda_{4}^{n}\right)\left\|\tilde{f}^{n}\right\|_{\mathcal{A}_{l}^{k}}+C_{2}\left(\lambda_{4}^{n}\right) \lambda_{k}^{n}\left\|\tilde{f}^{n}\right\|_{\mathcal{A}_{l}^{3}} .
$$

Similar to the Lemma 5.3 in [1], we also have

$$
\left\|\frac{f^{a}}{\partial_{\eta} u^{a 3}}\right\|_{\mathcal{A}_{l}^{k_{0}}\left([0, T] \times \mathbb{T} \times \mathbb{R}^{+}\right)} \leq C \varepsilon,
$$

because the construction of $\left(u^{a 3}, v^{a 3}\right)$ and the estimates in Proposition 4.1. Where $\varepsilon$ comes from the smallness of the integral interval of time. Then, as in [1, by studying estimates of $\tilde{f}^{n}$ and using an induction argument, we have 
Theorem 4.2 Under the same assumptions as those in Theorem 4.1, there exists a positive constant $C_{0}$ such that

$$
\left\|\omega^{n}\right\|_{\mathcal{A}_{l}^{k}} \leq C_{0} \varepsilon \theta_{n}^{\max }\{3-\tilde{k}, k-\tilde{k}\} \quad \triangle \theta_{n}
$$

holds for all $n \geq 0,0 \leq k \leq k_{0}$ and $\tilde{k} \geq 6$ here $\theta_{n}=\sqrt{\theta_{0}^{2}+n}$ and $\triangle \theta_{n}=\theta_{n+1}-\theta_{n}$.

Using the transformation (4.25), we can obtain

Corollary 4.1 Under the same assumptions as those in Theorem 4.2, the following estimates hold

$$
\left\|\delta u^{n}\right\|_{\mathcal{A}_{l}^{k}} \leq C \varepsilon \theta_{j}^{\max }\{3-\tilde{k}, k-\tilde{k}\}, \quad 0 \leq k \leq k_{0},
$$

and

$$
\left\|\delta v^{n}\right\|_{\mathcal{D}_{0}^{k}} \leq C_{1} \varepsilon \theta_{j}^{\max }\{3-\tilde{k}, k+1-\tilde{k}\}, \quad 0 \leq k \leq k_{0}-1
$$

\subsection{Existence to the nonlinear problem}

To show the existence of solution to the nonlinear boundary layer equations (1.1), we need to show the convergence of the iteration scheme (4.15)-(4.16). From this iteration, we know that the approximate solutions $\left(u^{n+1}, v^{n+1}\right)$ solve the following problem

$$
\left\{\begin{array}{l}
\mathcal{P}\left(u^{n+1}, v^{n+1}\right)=\left(1-S_{\theta_{n}}\right) \sum_{j=0}^{n} e_{j}+S_{\theta_{n}} e_{n}+\left(1-S_{\theta_{n}}\right) f^{a}, \\
\partial_{x}\left(\bar{\rho} u^{n+1}\right)+\partial_{\eta}\left(\bar{\rho} v^{n+1}\right)=-\bar{\rho}_{t}, \\
\left.u^{n+1}\right|_{\eta=0}=\left.v^{n+1}\right|_{\eta=0}=0, \quad \lim _{\eta \rightarrow+\infty} u^{n+1}=U(t, x), \\
\left.u^{n+1}\right|_{t=0}=u_{0}(x, \eta) .
\end{array}\right.
$$

From the estimates given in Corollary 4.1, we know that there exist functions $u \in \mathcal{A}_{l}^{\tilde{k}-2}$ and $v \in \mathcal{D}_{0}^{\tilde{k}-3}$, such that $u^{n}$ converges to $u$ in $\mathcal{A}_{l}^{\tilde{k}-2}$ and $v^{n}$ converges to $v$ in $\mathcal{D}_{0}^{\tilde{k}-3}$. In order to show the function pair $(u, v)$ is indeed a solution to the system (1.1), it suffices to show that the right hand side in equation (4.32) 1 converges to zero as $n$ tends to $+\infty$. Firstly, by using Lemma 4.1,

$$
\left\|\left(1-S_{\theta_{n}}\right)\left(f^{a}+\sum_{j=0}^{n} e_{j}\right)\right\|_{\mathcal{A}_{l}^{k}} \leq C \theta_{n}^{-1}\left(\left\|f^{a}\right\|_{\mathcal{A}_{l}^{k+1}}+\left\|\sum_{j=0}^{n} e_{j}\right\|_{\mathcal{A}_{l}^{k+1}}\right) .
$$

Then it suffices to show the $\left\|\sum_{j=0}^{n} e_{j}\right\|_{\mathcal{A}_{l}^{k+1}}$ converges. From the definition of $e_{j}=e_{j}^{1}+e_{j}^{2}$ given in (4.18)-(4.19), we have

$$
\left\|e_{j}^{1}\right\|_{\mathcal{A}_{l}^{k+1}} \leq C\left(\left\|\delta u^{j}\right\|_{L^{\infty}}\left\|\delta u^{j}\right\|_{\mathcal{A}_{l}^{k+2}}+\left\|\delta v^{j}\right\|_{L^{\infty}}\left\|\delta u^{j}\right\|_{\mathcal{A}_{l}^{k+2}}+\left\|\delta v^{j}\right\|_{\mathcal{D}_{0}^{k+2}}\left\|\delta u^{j}\right\|_{L_{\eta, l}^{2}\left(L_{t, x}^{\infty}\right)}\right)
$$




$$
\leq C \varepsilon^{2} \theta_{j}^{3-\tilde{k}+\max \{3-\tilde{k}, k+2-\tilde{k}\}}\left(\triangle \theta_{j}\right)^{2} \leq C \varepsilon^{2} \theta_{j}^{k+5-2 \tilde{k}} \triangle \theta_{j},
$$

for $k \leq \tilde{k}-5$. And

$$
\begin{aligned}
& \left\|e_{j}^{2}\right\|_{\mathcal{A}_{l}^{k+1}} \leq\left\|\left(1-S_{\theta_{j}}\right)\left(u^{j}-u^{a 3}\right) \partial_{\eta}\left(\delta v^{j}\right)\right\|_{\mathcal{A}_{l}^{k+1}}+2\left\|\frac{\bar{\rho}_{x}}{\bar{\rho}}\left(1-S_{\theta_{j}}\right)\left(u^{j}-u^{a 3}\right)\left(\delta u^{j}\right)\right\|_{\mathcal{A}_{l}^{k+1}} \\
& +\left\|\partial_{\eta}\left(\left(1-S_{\theta_{j}}\right)\left(v^{j}-v^{a 3}\right)\right)\left(\delta u^{j}\right)\right\|_{\mathcal{A}_{l}^{k+1}}+\left\|\left(\left(1-S_{\theta_{j}}\right)\left(v^{j}-v^{a 3}\right)\right) \partial_{\eta}\left(\delta u^{j}\right)\right\|_{\mathcal{A}_{l}^{k+1}} \\
& +\left\|\partial_{\eta}\left(\left(1-S_{\theta_{j}}\right)\left(u^{j}-u^{a 3}\right)\right)\left(\delta v^{j}\right)\right\|_{\mathcal{A}_{l}^{k+1}} \\
& \leq C\left(\left\|u^{j}-u^{a 3}\right\|_{L_{\eta, l}^{2}\left(L_{t, x}^{\infty}\right)}\left\|\delta v^{j}\right\|_{\mathcal{D}_{0}^{k+2}}+\left\|u^{j}-u^{a 3}\right\|_{\mathcal{A}_{l}^{k+1}}\left\|\partial_{\eta}\left(\delta v^{j}\right)\right\|_{L^{\infty}}\right. \\
& +\left\|u^{j}-u^{a 3}\right\|_{\mathcal{A}_{l}^{k+1} \|}\left\|\frac{\bar{\rho}_{x}}{\bar{\rho}} \delta u\right\|_{L^{\infty}}+\left\|u^{j}-u^{a 3}\right\|_{L^{\infty}}\left\|\frac{\bar{\rho}_{x}}{\bar{\rho}} \delta u\right\|_{\mathcal{A}_{l}^{k+1}}
\end{aligned}
$$

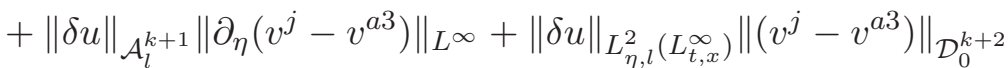

$$
\begin{aligned}
& +\|\delta u\|_{\mathcal{A}_{l}^{k+2}}\left\|\left(v^{j}-v^{a 3}\right)\right\|_{L^{\infty}}+\left\|\partial_{\eta} \delta u\right\|_{L_{\eta, l}^{2}\left(L_{t, x}^{\infty}\right)}\left\|\left(v^{j}-v^{a 3}\right)\right\|_{\mathcal{D}_{0}^{k+1}} \\
& +\left\|\delta v^{j}\right\|_{\mathcal{D}_{0}^{k+1}}\left\|\partial \eta\left(u^{j}-u^{a 3}\right)\right\|_{L_{\eta, l}^{2}\left(L_{t, x}^{\infty}\right)}+\left\|\delta v^{j}\right\|_{L^{\infty}}\left\|u^{j}-u^{a 3}\right\|_{\left.\mathcal{A}_{l}^{k+2}\right)} \\
& \leq C \varepsilon^{2} \theta_{j}^{k+3-\tilde{k}} \triangle \theta_{j},
\end{aligned}
$$

for $k \leq \tilde{k}-5$. Thus, we get that

$$
\sum_{j=0}^{+\infty}\left\|e_{j}\right\|_{\mathcal{A}_{l}^{k}} \leq C \sum_{j=0}^{+\infty} \theta_{j}^{k+3-\tilde{k}} \triangle \theta_{j} \leq C C_{0}
$$

for $k \leq \tilde{k}-5$.

Therefore, the right hand side of (4.32) 1 tends to zero as $n$ tends to $+\infty$. The uniqueness

of classical solutions to (1.1) can be proved as in [1. Then we complete the proof of Theorem 1.1 .

Acknowledgements: The first author's research was supported in part by National Natural Science Foundation of China (NNSFC) under Grants No. 10971134, No. 11031001 and No. 91230102. The second author is supported by NSFC No.11171213, Shanghai Rising Star Program No.12QA1401600. The last author's research was supported by the General Research Fund of Hong Kong, CityU No. 103713.

\section{References}

[1] Alexander, R., Wang, Y-G, Xu, C-J., Yang, T., Well posedness of the Prandtl eqauation in Sobolev spaces, to appear in J. Amer. Math. Soc., arXiv:1203.5991v1.

[2] Caflisch, R.E., Sammartino, M., Existence and singularities for the Prandtl boundary layer equations, Z. Angew. Math. Mech., 80(2000), 733-744.

[3] E, W., Boundary layer theory and the zero-viscosity limit of the Navier-Stokes equation. Acta Math. Sin. (Engl. Ser.) 16(2000), 207-218. 
[4] E, W., Engquist, B., Blow up of solutions of the unsteady Prandtl equation, Comm. Pure Appl. Math., 50(1997), 1287-1293.

[5] Gerard-Varet, D., Dormy, E., On the ill-posedness of the Prandtl equation, J. Amer. Math. Soc., 23(2010), 591-609.

[6] Gerard-Varet, D., Nguyen, T., Remarks on the ill-posedness of the Prandtl equation, preprint 2010. arXiv:1008.0532v1.

[7] Grenier, E., On the nonlinear instability of Euler and Prandtl equations. Comm. Pure Appl. Math. 53(2000), 1067-1091.

[8] Guo, Y., Nguyen, T., A note on the Prandtl boundary layers, Comm. Pure Appl. Math. 64(2011), 1416-1438.

[9] Maekawa,Y., On the inviscid limit problem of the vorticity equations for viscous incompressible flows in the half plane, preprint (2012).

[10] Métivier,G., Small Viscosity and Boundary Layer Methods. Theory, Stability Analysis, and Applications. Modeling and Simulation in Science, Engineering and Technology. Birkhauser Boston, Inc., Boston, MA, 2004. xxii+194 pp

[11] Masmoudi N., Wong T-K, Local-in-time existence and uniqueness of solutions to the Prandtl equations by energy methods, arXiv:1206.3629, (2012).

[12] Williams, M., Wang, Y-G, The inviscid limit and stability of characteristic boundary layers for the compressible Navier-Stokes equations with Navier-friction boundary conditions, Ann. Inst. Fourier 62 no.6(2012), 2257-2314.

[13] Moser, J., A new technique for the construction of solutions of nonlinear differential equations, Proc. Nat. Acad. Sci., 47(1961), 1824-1831.

[14] Nash, J.: The imbedding problem for Riemannian manifolds, Ann. of Math., 63(1956), $20-63$.

[15] Oleinik, O. A., The Prandtl system of equations in boundary layer theory. Soviet Math Dokl, 4(1963), 583-586.

[16] Oleinik, O. A., Samokhin, V. N., Mathematical Models in Boundary Layers Theory. Chapman and Hall/CRC, 1999.

[17] Prandtl, L., Uber flüssigkeits-bewegung bei sehr kleiner reibung. Verhandlungen des III. Internationlen Mathematiker Kongresses, Heidelberg. Teubner, Leipzig, (1904), 484-491.

[18] Wang, Y-G., Xin, Z-P., Wang, X-P., Boundary layers in incompressible navier-stokes equations with navier boundary conditions for the vanishing viscosity limit. Comm. Math. Sci., 8(2010), 965-998.

[19] Xiao, Y.L., Xin, Z.P., On the vanishing viscosity limit for the 3D Navier-Stokes equations with a slip boundary condition. Comm. Pure Appl. Math.60(7), (2007)1027-1055. 
[20] Xiao, Y.L., Xin, Z.P., A new boundary condition for the three-dimensional Navier-Stokes equation and the vanishing viscosity limit. J. Math. Phys. 53 (2012).

[21] Xin, Z.P., Yanagisawa T., Zero-viscosity limit of the linearized navier-stokes equations for a compressible viscous fluid in the half-plane. Comm. Pure Appl. Math., 52(1999), 479-541.

[22] Xin, Z-P., Zhang, L., On the global existence of solutions to the Prandtl system, Adv. Math., 181(2004), 88-133. 